\title{
Lattès Maps and Combinatorial Expansion
}

\author{
by \\ Qian Yin \\ A dissertation submitted in partial fulfillment \\ of the requirements for the degree of \\ Doctor of Philosophy \\ (Mathematics) \\ in The University of Michigan
}

2011

Doctoral Committee:

Professor Mario Bonk, University of California, Los Angeles, Co-Chair

Professor Michael E. Zieve, Co-Chair

Professor Mattias Jonsson

Associate Professor James P. Tappenden

Professor Dennis P. Sullivan, Graduate School and University Center of the City University of New York and State University of New York at Stony Brook 
(C) $\quad \frac{\text { Qian Yin }}{\text { All Rights }} \begin{gathered}2011 \\ \text { Reserved }\end{gathered}$ 
to Mama and Baba 


\section{ACKNOWLEDGEMENTS}

I am deeply indebted to my advisor Mario Bonk for introducing me to and teaching me about the subject of Thurston maps, for his countless hours spent sharing his mathematical wisdom and enthusiasm with me and for providing a perfect role model as a 'precise mathematician'. I am especially grateful for his patience and encouragement. I am enormously grateful to Michael Zieve for his brilliant suggestions and his careful reading of this thesis, and to Dennis Sullivan for his valuable conversations and for sharing his mathematical insights during lunches at Tiger Lily. I also benefited enormously from the brilliance of Dick Canary, Peter Duren, Lizhen Ji, Mattias Jonsson, Pekka Pankka, Gopal Prasad, Peter Scott, Ralf Spatzier, Karen Smith and Juan Souto. I would also like to thank James Tappenden for serving on my committee.

I would like to thank Alan Stapledon for helping me get through the difficult times, for all the emotional support and especially for his patience listening to my reasonable and unreasonable complaints. I wish to thank all my friends in graduate school, in particular, Michelle Lee, Eugene Eisenstein, Dan Hernandez, Xueying Hu, Ricardo Portilla, Yefeng Shen, Emily Witt, Nina White and Crystal Zeager for providing a stimulating and fun environment in which to learn and grow. I am also indebted to my mentors back in China, in particular, Fang Li, Xingjiang Lu, Shiuchun Wong and Yongcheng Yin.

Lastly, and most importantly, I would like to thank my Mama and Baba for their 
unconditional love and unshakable support. To them I dedicate this thesis. 


\section{TABLE OF CONTENTS}

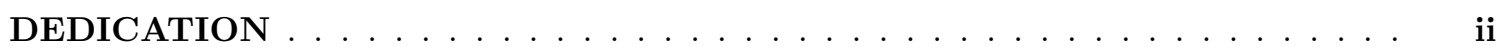

ACKNOWLEDGEMENTS ...................... i ii

\section{CHAPTER}

I. Introduction $\ldots \ldots \ldots \ldots \ldots \ldots \ldots$

1.1 History . . . . . . . . . . . . . . . . . . . . 1

1.2 Thurston Maps . . . . . . . . . . . . . . . . . . . . . . . . 4

1.3 Summary of Results . . . . . . . . . . . . . . . . . . 5

II. Preliminaries . . . . . . . . . . . . . . . . . . . . . . . . . . 12

2.1 Expanding Thurston maps and Cell Decompositions . . . . . . . . . . . . 12

2.2 Gromov Hyperbolic Spaces . . . . . . . . . . . . . . . . . . . . 20

III. Lattès Maps and Combinatorial Expansion . . . . . . . . . . . . . . . . . . . . 24

3.1 Lattès and Lattès-type Maps . . . . . . . . . . . . . . . . . . . . . . . . . . 24

3.2 Combinatorial Expansion Factor and $D_{n} \ldots \ldots \ldots \ldots$. . . . . . . . . . . . . . 33

3.3 Existence of the Visual Metric . . . . . . . . . . . . . . . . . . . 40

3.4 The Sufficiency of the Conditions . . . . . . . . . . . . . . . . . . 55

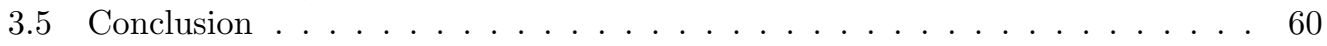

IV. Thurston Maps and Asymptotic Upper Curvature . . . . . . . . . . . . . 62

4.1 Tile Graphs . . . . . . . . . . . . . . . . . . . . . . 62

4.2 Asymptotic Upper Curvature . . . . . . . . . . . . . . . . . . . . 75

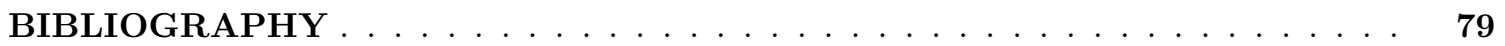




\section{CHAPTER I}

\section{Introduction}

\subsection{History}

A rational map $f: \widehat{\mathbb{C}} \rightarrow \widehat{\mathbb{C}}$ is a special type of analytic map on the Riemann sphere $\widehat{\mathbb{C}}=\mathbb{C} \cup\{\infty\}$. It can be written as a quotient of two relatively prime complex polynomials $p(z)$ and $q(z)$, with $q(z) \neq 0$,

$$
f(z)=\frac{p(z)}{q(z)}=\frac{a_{0} z^{m}+\ldots+a_{m}}{b_{0} z^{l}+\ldots+b_{l}}
$$

where $a_{i}, b_{j} \in \mathbb{C}$ for $i=0, \ldots, m$ and $j=0, \ldots, l$. The fundamental problem in dynamics is to understand the behavior of the iterates of $f$,

$$
f^{n}(z):=\underbrace{f \circ f \circ \cdots \circ f}_{n \text { factors }}(z) .
$$

The study of the dynamics of rational maps was originated in 1917 by Pierre Fatou and Gaston Julia, who developed the foundations of complex dynamics. In particular, they applied Montel's theory of normal families to develop the fundamental theory of iteration (see [8] and [11]). Their work was more or less forgotten for over half a century. Then Benoit Mandelbrot rekindled interest in the field in the 1970s by generating beautiful and intriguing graphic images that naturally appear under iteration of rational maps through his computer experiments (see [14] and [15]). In recent years, the study of dynamics of rational maps has attracted 
considerable interest, not only because complex dynamics itself is an intriguing and rich subject, but also because of its links to other branches of mathematics, such as quasi-conformal mappings, Kleinian groups, potential theory and algebraic geometry. For instance, the study of the dynamical systems arising from polynomials and those that arise from Kleinian groups that depend on holomorphic motions are connected to the dictionary introduced by Sullivan (see [21]), which led to his seminal work on the non-existence of wandering domains for rational maps.

Given a rational map $f: \widehat{\mathbb{C}} \rightarrow \widehat{\mathbb{C}}$, the degree $\operatorname{deg}(f)$ of $f$ is the maximal degree of the polynomials $p(z)$ and $q(z)$ as in equation (1.1). The degree of $f$ can also be defined topologically as the cardinality of the preimage over a generic (non-critical) value.

A rational map $f$ with $\operatorname{deg}(f)>1$ can have both expanding and contracting features. The tension between these two features makes the dynamics of rational maps involved and interesting. We will assume that the rational map $f$ has $\operatorname{deg}(f)>$ 1 from now on. A point $z \in \widehat{\mathbb{C}}$ is periodic if $f^{n}(z)=z$ for some $n \in \mathbb{N}$. In this case, it is called

$$
\begin{array}{ll}
\text { attracting } & \text { if }\left|\left(f^{n}\right)^{\prime}(z)\right|<1 ; \\
\text { indifferent } & \text { if }\left|\left(f^{n}\right)^{\prime}(z)\right|=1 ; \\
\text { repelling } & \text { if }\left|\left(f^{n}\right)^{\prime}(z)\right|>1 .
\end{array}
$$

For example, if we let $f(z)=z^{2}$, then $z=0$ is an attracting periodic point of $f$, and $f$ is contracting near $0 ; z=1$ is a repelling periodic point, and $f$ is expanding near 1.

The Julia set $J(f)$ of $f$ is the closure of the set of repelling periodic points. It is also the smallest closed set containing at least three points which is completely invariant under $f^{-1}$. For the example $f(z)=z^{2}$, the Julia set of $f$ is the unit circle. 
The complement $F(f)=\widehat{\mathbb{C}} \backslash J(f)$ of the Julia set, called the Fatou set, is the largest open set such that the iterates of $f$ restricted to it form a normal family. The Julia set and Fatou set are both invariant under $f$ and $f^{-1}$.

The postcritical set post $(f)$ of $f$ is the closure of the forward orbits of the critical points

$$
\operatorname{post}(f)=\overline{\bigcup_{n \geq 1}\left\{f^{n}(c): c \in \operatorname{crit}(f)\right\}} .
$$

The postcritical set plays a crucial role in terms of understanding the expanding and contracting features of a rational map. If the postcritical set post $(f)$ is finite, we say that the map $f$ is postcritically finite. In the postcritically finite case,

$$
\operatorname{post}(f)=\bigcup_{n \geq 1}\left\{f^{n}(c): c \in \operatorname{crit}(f)\right\}
$$

In 1918, Samuel Lattès described a special class of rational maps which have a simultaneous linearization for all of their periodic points (see [12]). This class of maps is named after Lattès, even though similar examples had been studied by Ernst Schröder much earlier (see [20]). A Lattès map $f: \widehat{\mathbb{C}} \rightarrow \widehat{\mathbb{C}}$ is a rational map that is obtained from a finite quotient of a conformal torus endomorphism, i.e., the map $f$ satisfies the following commutative diagram:

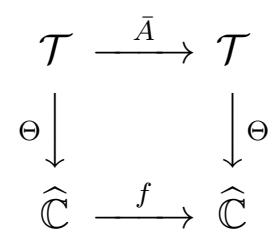

where $\bar{A}$ is a map of a torus $\mathcal{T}$ that is a quotient of an affine map of the complex plane, and $\Theta$ is a finite-to-one holomorphic map. Lattès maps were the first examples of rational maps whose Julia set is the whole sphere $\widehat{\mathbb{C}}$, and the postcritical set of a Lattès map is finite. More importantly, Lattès maps play a central role as exceptional examples in complex dynamics. We will discuss this further in the following section. 


\subsection{Thurston Maps}

Observing that much information about the dynamics of a rational map can be deduced from the postcritical set, Thurston introduced a topological analog of a postcritically finite rational map, now known as a Thurston map (see [7]). A Thurston map $f: \mathbb{S}^{2} \rightarrow \mathbb{S}^{2}$ is a branched covering map with finite postcritical set post $(f)$. Thurston gave a characterization of Lattès maps among Thurston maps in terms of the angles of cone points on associated orbifolds. More precisely, for any Thurston map $f$, there exists a unique smallest function $\nu_{f}$ among functions $\nu: \mathbb{S}^{2} \rightarrow \mathbb{N} \cup\{\infty\}$ such that

$$
\nu(p) \operatorname{deg}_{f}(p) \mid \nu(f(p))
$$

for all $p \in \mathbb{S}^{2}$. Thurston associated an orbifold $O_{f}=\left(\mathbb{S}^{2}, \nu_{f}\right)$ to a Thurston map $f$ using the function $\nu_{f}$ (see [7]). For each $p \in \mathbb{S}^{2}$ with $v_{f}(p) \neq 1$, the point $p$ is a cone point with cone angle $2 \pi / v_{f}(p)$. Let

$$
\chi\left(O_{f}\right)=2-\sum_{p \in \operatorname{post}(f)}\left(1-\frac{1}{\nu_{f}(p)}\right) .
$$

If $\chi\left(O_{f}\right)=0$, we say that the orbifold $O_{f}$ is parabolic. The orbifold $O_{f}$ of a Lattès map is parabolic, and the cardinality of the postcritical set can only be 3 or 4 . Lattès maps with \# post $(f)=4$ are also referred to as flexible Lattès maps.

The notion of an expanding Thurston map was introduced in [3] as a topological analog of a postcritically finite rational map whose Julia set is the whole sphere $\widehat{\mathbb{C}}$. Roughly speaking, a Thurston map is called expanding if all the connected components of the preimage under $f^{-n}$ of any open Jordan region disjoint from post $(f)$ become uniformly small as $n$ tends to infinity. We refer the reader to Definition II.1 for a more precise statement. A related and more general notion of expanding Thurston 
maps was introduced in [9]. Lattès maps are among the simplest examples of expanding Thurston maps.

Lattès maps are distinguished among all rational maps in various ways. For instance, Lattès maps are the only rational maps for which the measure of maximal entropy is absolutely continuous with respect to Lebesgue measure (see [23]).

Many different characterizations of Lattès maps have been attempted (e.g. [17]). For example, a fundamental conjecture in complex dynamics states that the flexible Lattès maps are the only rational maps that admit an "invariant line field" on their Julia set. The significance of this conjecture is demonstrated by a theorem of Mañé, Sad and Sullivan (see [16]). It states that if the fundamental conjecture above is true, then hyperbolic maps are dense among rational maps. We refer the reader to [18] for a nice exposition on Lattès maps.

\subsection{Summary of Results}

Let $f$ be an expanding Thurston map, and let $\mathcal{C}$ be a Jordan curve containing post $(f)$. The Jordan Curve Theorem implies that $\mathbb{S}^{2} \backslash \mathcal{C}$ has precisely two connected components, whose closures are called 0-tiles. The closure of each connected component of the preimage of $\mathbb{S}^{2} \backslash \mathcal{C}$ under $f^{n}$ is called an $n$-tile. In Section 5 of [3], it is proved that the collection of all $n$-tiles gives a cell decomposition of $\mathbb{S}^{2}$. The points in $\operatorname{post}(f)$ divide $\mathcal{C}$ into several subarcs. Let $D_{n}=D_{n}(f, \mathcal{C})$ be the minimum number of $n$-tiles needed to join two of these subarcs that are non-adjacent (see Definition III.9 and (4.2)). For any Thurston map $f$, there exists $C>0$ such that

$$
D_{n} \leq C(\operatorname{deg} f)^{n / 2}
$$

for all $n>0$ (see Proposition III.22). For Lattès maps, an inequality as in (1.4) is also true in the opposite direction (see Proposition III.18). One of my main results 
of this thesis asserts that, in fact, this inequality characterizes Lattès maps among expanding Thurston maps with no periodic critical points (see Theorem III.41).

Theorem I.1. A map $f: \mathbb{S}^{2} \rightarrow \mathbb{S}^{2}$ is topologically conjugate to a Lattès map if and only if the following conditions hold:

- $f$ is an expanding Thurston map;

- $f$ has no periodic critical points;

- there exists $c>0$ such that $D_{n} \geq c(\operatorname{deg} f)^{n / 2}$ for all $n>0$.

Let $f$ be an expanding Thurston map. Even though $D_{n}=D_{n}(f, \mathcal{C})$ depends on the Jordan curve $\mathcal{C}$, its growth rate is independent of the $\mathcal{C}$. So the limit

$$
\Lambda_{0}(f)=\lim _{n \rightarrow \infty}\left(D_{n}(f, \mathcal{C})\right)^{1 / n}
$$

exists and only depends on the map $f$ itself (see [3, Prop. 17.1]). We call this limit $\Lambda_{0}(f)$ the combinatorial expansion factor of $f$. This quantity $\Lambda_{0}(f)$ is invariant under topological conjugacy and multiplicative in the sense that $\Lambda_{0}(f)^{n}$ is the combinatorial expansion factor of $f^{n}$. Inequality (1.4) implies that

$$
\Lambda_{0}(f) \leq(\operatorname{deg} f)^{1 / 2}
$$

The combinatorial expansion factor is closely related to the notion of visual metrics and their expansion factors. Every expanding Thurston map $f: \mathbb{S}^{2} \rightarrow \mathbb{S}^{2}$ induces a natural class of metrics on $\mathbb{S}^{2}$, called visual metrics (see Definition II.11), and each visual metric $d$ has an associated expansion factor $\Lambda>1$. This visual metric is essentially characterized by the geometric property that the diameter of an $n$-tile is about $\Lambda^{-n}$, and the distance between two disjoint $n$-tiles is at least about $\Lambda^{-n}$. The supremum of the expansion factors of all visual metrics is equal to the combinatorial 
expansion factor $\Lambda_{0}$ (see [3, Theorem 1.5]). For Lattès maps, the supremum is obtained. In general, the supremum is not obtained. Examples are the Lattès-type maps that are not Lattès maps (see Section 3.1). We will show in Proposition III.31 that Theorem I.1 remains true if we replace the third condition by the requirement that there exists a visual metric on $\mathbb{S}^{2}$ with expansion factor $\Lambda=(\operatorname{deg} f)^{1 / 2}$.

Here we outline the sufficiency of the three conditions in Theorem I.1. These three conditions imply the existence of a visual metric $d$ on $\mathbb{S}^{2}$ with expansion factor $\Lambda=(\operatorname{deg} f)^{1 / 2}$ (see Proposition III.31). The way that we construct the visual metric uses the idea that any quasi-visual metric can be modified to be a visual metric. The existence of this visual metric implies that $\left(\mathbb{S}^{2}, d\right)$ is Ahlfors 2-regular, which means any ball with radius $r$ has Hausdorff 2-measure roughly $r^{2}$ (see Proposition III.33). Using this 2-regularity together with the linear local connectivity condition of $\left(\mathbb{S}^{2}, d\right)$, we obtain that $\left(\mathbb{S}^{2}, d\right)$ is quasisymmetrically equivalent to the Riemann sphere $\widehat{\mathbb{C}}$ by [2, Theorem 1.1] (see Proposition III.33). We deduce that $f$ is topologically conjugate to a rational map from the quasisymmetrical equivalence of $\left(\mathbb{S}^{2}, d\right)$ to the Riemann sphere $\widehat{\mathbb{C}}$ (see Proposition III.35). Now we can focus on the rational maps with three conditions satisfied in Theorem I.1. In order to invoke the characterization of Lattès maps among rational maps by [17], we need that the Hausdorff measure with respect to the visual metric and with respect to the standard chordal metric $d$ on $\widehat{\mathbb{C}}$ are essentially the same. This follows from [10, Corollary 7.13], and it implies that the dimension of Lebesgue measure with respect to the visual metric $d$ is equal to 2 (see Theorem III.39). We conclude that the map $f$ is topologically conjugate to a Lattès map.

We define Lattès-type maps so as to include non-rational maps that are quotients of affine maps and share many desired properties of Lattès maps. Comparing with 
diagram 1.2, we have a commutative diagram

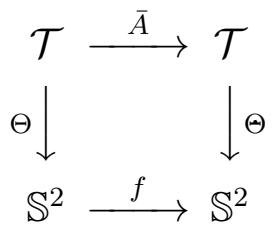

where $\Theta$ is essentially the same $\Theta$ as in diagram 1.2, and we require $\bar{A}$ to be a quotient of an affine map on the real plane rather than the complex plane. A map $f: \mathbb{S}^{2} \rightarrow \mathbb{S}^{2}$ obtained by the above commutative diagram is called a Lattès-type map (see Definition III.2). If a Lattès-type map is rational, then the map is a Lattès map.

Lattès-type maps are examples of expanding Thurston maps, and they have the same orbifold structures as Lattès maps (see Proposition III.16).

Proposition I.2. Let $f$ be a Lattès-type map with orbifold type $(2,2,2,2)$. Let $A$ be its corresponding linear map from $\mathbb{R}^{2}$ to $\mathbb{R}^{2}$ and let $\wp: \mathbb{R}^{2} \rightarrow \mathbb{S}^{2}$ be the Weierstrass function with the lattice $2 \mathbb{Z}^{2}$. We have

$$
\frac{1}{\left\|A^{-n}\right\|_{\infty}} \leq D_{n}(f, \mathcal{C}) \leq \frac{1}{\left\|A^{-n}\right\|_{\infty}}+1
$$

where the Jordan curve $\mathcal{C}$ is the image of the boundary of the unit square $[0,1] \times[0,1]$ under $\wp$.

Here $\|B\|_{\infty}$ denotes the operator norm of a linear map $B$ on $\mathbb{R}^{2}$ with respect to the $\ell^{\infty}$-norm. As a corollary of this proposition and equation (1.5), we have the following result (see Corollary III.17).

Corollary I.3. Let $f$ be a Lattès-type map with orbifold type $(2,2,2,2)$, and let $A$ be the corresponding linear map from $\mathbb{R}^{2}$ to $\mathbb{R}^{2}$. Then the combinatorial expansion factor $\Lambda_{0}(f)$ equals the minimum absolute value of the eigenvalues of $A$.

A geodesic metric space $(X, d)$ is called a Gromov hyperbolic space if every geodesic triangle in it is "very thin". It can also defined in terms of Gromov products. For 
any points $x, y, p \in X$, the Gromov product $(x, y)_{p}$ of $x$ and $y$ with respect to the base point $p$ is defined as

$$
(x, y)_{p}:=\frac{1}{2}[d(x, p)+d(y, p)-d(x, y)] .
$$

The space $X$ is called $\delta$-hyperbolic (or Gromov hyperbolic) for some $\delta \geq 0$ if there exists a base point $p \in X$ such that for all $x, y, z \in X$, we have

$$
(x, y)_{p} \geq \min \left\{(x, z)_{p},(z, y)_{p}\right\}-\delta .
$$

We construct a graph $\mathcal{G}=\mathcal{G}(f, \mathcal{C})$ by letting the tiles in the cell decompositions of $(f, \mathcal{C})$ be vertices of $\mathcal{G}$. There is an edge between the two vertices $X^{n}, Y^{m} \in V$, denoted $X^{n} \sim Y^{m}$ if as underlying tiles

$$
|n-m| \leq 1 \text { and } X^{n} \cap Y^{m} \neq \emptyset .
$$

It turns out that the graph $\mathcal{G}$ with the path metric is a Gromov hyperbolic space (see Theorem IV.5).

Theorem I.4. Let $f: \mathbb{S}^{2} \rightarrow \mathbb{S}^{2}$ be an expanding Thurston map without periodic critical points and let $\mathcal{C} \subset \mathbb{S}^{2}$ be a Jordan curve containing post $(f)$. Then the graph $\mathcal{G}(f, \mathcal{C})$ equipped with the path metric $\eta$ is a Gromov hyperbolic space.

There is a natural boundary at infinity of a Gromov hyperbolic space. Roughly speaking, the boundary at infinity is the set of equivalence classes of geodesic rays in the Gromov hyperbolic space. It can also be equipped with a Gromov product by taking infimum of the infimum limit of the Gromov product along all the geodesic rays among the corresponding equivalence classes. A visual metric $\rho$ on the boundary at infinity of a Gromov hyperbolic space is a metric that has a bounded ratio

$$
\rho\left(\xi, \xi^{\prime}\right) / \Lambda^{-\left(\xi, \xi^{\prime}\right)_{p}}
$$


for some fixed $\Lambda>1$ and for all points $\xi$ and $\xi^{\prime}$ on the boundary.

In Proposition IV.7, we show that the boundary at infinity $\partial_{\infty} \mathcal{G}=\partial_{\infty} \mathcal{G}(f, \mathcal{C})$ can be identified with $\mathbb{S}^{2}$; under this identification, a metric $d$ is a visual metric on $\mathbb{S}^{2}$ with respect to the expanding Thurston map $f$ if and only if $d$ is a visual metric (in the sense of Gromov hyperbolic space) on $\partial_{\infty} \mathcal{G}$. We deduce that for any Jordan curves $\mathcal{C}$ and $\mathcal{C}^{\prime}$ containing post $(f)$, the classes of visual metrics on $\partial_{\infty} \mathcal{G}(f, \mathcal{C})$ and $\partial_{\infty} \mathcal{G}\left(f, \mathcal{C}^{\prime}\right)$ can also be identified (see Corollary IV.8).

In [1], the asymptotic upper curvature of a Gromov hyperbolic space is introduced. It is the analogue of sectional curvature on Riemannian manifolds. Fix $\kappa \in[-\infty, 0)$. We call a metric space $X$ an $A C_{u}(\kappa)$-space if there exists $p \in X$ and a constant $c \geq 0$ such that for all $x, x^{\prime} \in X$ and all finite sequences $x_{0}=x, x_{1}, \ldots, x_{n}=x^{\prime}$ in $X$,

$$
\left(x, x^{\prime}\right)_{p} \geq \min _{i=1, \ldots, n}\left(x_{i-1}, x_{i}\right)_{p}-\frac{1}{\sqrt{-\kappa}} \log n-c .
$$

Here we use the convention $1 / \sqrt{\infty}=0$. We call

$$
K_{u}(X):=\inf \left\{\kappa \in[-\infty, 0): X \text { is an } \mathrm{AC}_{u}(\kappa) \text {-space }\right\}
$$

the asymptotic upper curvature of $X$. It is invariant under rough-isometry.

For any Jordan curves $\mathcal{C}$ and $\mathcal{C}^{\prime}$ containing post $(f)$, the metric spaces $\mathcal{G}=\mathcal{G}(f, \mathcal{C})$ and $\mathcal{G}^{\prime}=\mathcal{G}\left(f, \mathcal{C}^{\prime}\right)$ are rough-isometric (see Proposition IV.6). Hence we may define the asymptotic upper curvature $K_{u}(f)$ of an expanding Thurston map $f$ as

$$
K_{u}(f):=K_{u}(\mathcal{G}(f, \mathcal{C})),
$$

where $\mathcal{C} \subseteq \mathbb{S}^{2}$ is any Jordan curve containing post $(f)$. Using the notation above, we have the following theorem.(see Theorem IV.9).

Theorem I.5. Let $f: \mathbb{S}^{2} \rightarrow \mathbb{S}^{2}$ be an expanding Thurston map without periodic 
critical points. The asymptotic upper curvature of $f$ satisfies

$$
K_{u}(f) \geq-\frac{1}{4} \log ^{2}(\operatorname{deg} f)
$$

The tile graph $\mathcal{G}=\mathcal{G}(f)$ is an $A C_{u}(\kappa)$-space with

$$
\kappa=-\frac{1}{4} \log ^{2}(\operatorname{deg} f)
$$

if and only if the map $f$ is topologically conjugate to a Lattès map. 


\section{CHAPTER II}

\section{Preliminaries}

\subsection{Expanding Thurston maps and Cell Decompositions}

In this section we review some definitions and facts on expanding Thurston maps. We refer the reader to Section 3 in [3] for more details. We write $\mathbb{N}$ for the set of positive integers, and $\mathbb{N}_{0}$ for the set of non-negative integers. We denote the identity map on $\mathbb{S}^{2}$ by $\operatorname{id}_{\mathbb{S}^{2}}$.

Let $\mathbb{S}^{2}$ be a topological 2-sphere with a fixed orientation. A continuous map $f: \mathbb{S}^{2} \rightarrow \mathbb{S}^{2}$ is called a branched covering map over $\mathbb{S}^{2}$ if $f$ can be locally written as

$$
z \mapsto z^{d}
$$

under certain orientation-preserving coordinate changes of the domain and range. More precisely, we require that for any point $p \in \mathbb{S}^{2}$, there exists some integer $d>0$, an open neighborhood $U_{p} \subseteq \mathbb{S}^{2}$ of $p$, an open neighborhood $V_{q} \subseteq \mathbb{S}^{2}$ of $q=f(p)$, and orientation-preserving homeomorphism

$$
\phi: U_{p} \rightarrow U \subseteq \mathbb{C}
$$

and

$$
\psi: V_{p} \rightarrow V \subseteq \mathbb{C}
$$


with $\phi(p)=0$ and $\psi(q)=0$ such that

$$
\left(\psi \circ f \circ \phi^{-1}\right)(z)=z^{d}
$$

for all $z \in U$. The positive integer $d=\operatorname{deg}_{f}(p)$ is called the local degree of $f$ at $p$ and only depends on $f$ and $p$. A point $p \in \mathbb{S}^{2}$ is called a critical point of $f$ if $\operatorname{deg}_{f}(p) \geq 2$, and a point $q$ is called critical value of $f$ if there is a critical point in its preimage $f^{-1}(q)$. If $f$ is a branched covering map of $\mathbb{S}^{2}, f$ is open and surjective. There are only finitely many critical points of $f$ and $f$ is finite-to-one due to the compactness of $\mathbb{S}^{2}$. Hence, $f$ is a covering map away from the critical points in the domain and critical values in the range. The degree $\operatorname{deg}(f)$ of $f$ is the cardinality of the preimage over a non-critical value. In addition, we have

$$
\operatorname{deg}(f)=\sum_{p \in f^{-1}(q)} \operatorname{deg}_{f}(p)
$$

for every $q \in \mathbb{S}^{2}$.

For $n \in \mathbb{N}$, we denote the $n$-th iterate of $f$ as

$$
f^{n}=\underbrace{f \circ f \circ \cdots \circ f}_{n \text { factors }} .
$$

We also set $f^{0}=\mathrm{id}_{\mathbb{S}^{2}}$.

If $f$ is a branched cover of $\mathbb{S}^{2}$, so is $f^{n}$, and

$$
\operatorname{deg}\left(f^{n}\right)=\operatorname{deg}(f)^{n}
$$

Let $\operatorname{crit}(f)$ be the set of all the critical points of $f$. We define the set of postcritical points of $f$ as

$$
\operatorname{post}(f)=\bigcup_{n \in \mathbb{N}}\left\{f^{n}(c): c \in \operatorname{crit}(f)\right\} .
$$

We call a map $f$ postcritically-finite if the cardinality of post $(f)$ is finite. Notice that $f$ is postcritically-finite if and only if there is some $n \in \mathbb{N}$ for which $f^{n}$ is postcritically-finite. 
Let $\mathcal{C} \subseteq \mathbb{S}^{2}$ be a Jordan curve containing post $(f)$. We fix a metric $d$ on $\mathbb{S}^{2}$ that induces the standard metric topology on $\mathbb{S}^{2}$. Denote by mesh $(f, n, \mathcal{C})$ the supremum of the diameters of all connected components of the set $f^{-n}\left(\mathbb{S}^{2} \backslash \mathcal{C}\right)$.

Definition II.1. A branched covering map $f: \mathbb{S}^{2} \rightarrow \mathbb{S}^{2}$ is called a Thurston map if $\operatorname{deg}(f) \geq 2$ and $f$ is postcritically-finite. A Thurston map $f: \mathbb{S}^{2} \rightarrow \mathbb{S}^{2}$ is called expanding if there exists a Jordan curve $\mathcal{C} \subseteq \mathbb{S}^{2}$ with $\mathcal{C} \supseteq \operatorname{post}(f)$ and

$$
\lim _{n \rightarrow \infty} \operatorname{mesh}(f, n, \mathcal{C})=0 .
$$

The relation (2.1) is a topological property, as it is independent of the choice of the metric, as long as the metric induces the standard topology on $\mathbb{S}^{2}$. Lemma 8.1 in [3] shows that if the relation (2.1) is satisfied for one Jordan curve $\mathcal{C}$ containing post $(f)$, then it holds for every such curve. One can essentially show that a Thurston map is expanding if and only if all the connected components in the preimage under $f^{-n}$ of any open Jordan region not containing post $(f)$ become uniformly small as $n$ goes to infinity.

The following theorem (Theorem 1.2 in [3]) says that there exists an invariant Jordan curve for some iterates of $f$.

Theorem II.2. If $f: \mathbb{S}^{2} \rightarrow \mathbb{S}^{2}$ is an expanding Thurston map, then for some $n \in \mathbb{N}$ there exists a Jordan curve $\mathcal{C} \subseteq \mathbb{S}^{2}$ containing post $(f)$ such that $\mathcal{C}$ is invariant under $f^{n}$, i.e., $f^{n}(\mathcal{C}) \subseteq \mathcal{C}$

Recall that an isotopy $H$ between two homeomorphisms is a homotopy so that at each time $t \in[0,1]$, the map $H_{t}$ is a homeomorphism. An isotopy $H$ relative to a set $A$ is an isotopy satisfying

$$
H_{t}(a)=H_{0}(a)=H_{1}(a)
$$

for all $a \in A$ and $t \in[0,1]$. 
Definition II.3. Consider two Thurston maps $f: \mathbb{S}^{2} \rightarrow \mathbb{S}^{2}$ and $g: \mathbb{S}_{1}^{2} \rightarrow \mathbb{S}_{1}^{2}$, where $\mathbb{S}^{2}$ and $\mathbb{S}_{1}^{2}$ are 2-spheres. We call the maps $f$ and $g$ (Thurston) equivalent if there exist homeomorphisms $h_{0}, h_{1}: \mathbb{S}^{2} \rightarrow \mathbb{S}_{1}^{2}$ that are isotopic relative to post $(f)$ such that $h_{0} \circ f=g \circ h_{1}$. We call the maps $f$ and $g$ topologically conjugate if there exists a homeomorphism $h: \mathbb{S}^{2} \rightarrow \mathbb{S}_{1}^{2}$ such that $h \circ f=g \circ h$.

For equivalent Thurston maps, we have the following commutative diagram

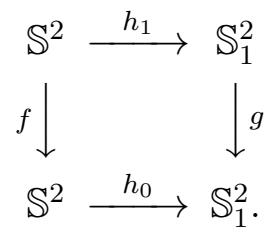

If $f: \mathbb{S}^{2} \rightarrow \mathbb{S}^{2}$ is an expanding Thurston map and $g: \mathbb{S}_{1}^{2} \rightarrow \mathbb{S}_{1}^{2}$ is topologically conjugate to $f$, then $g$ is also expanding. If $f$ and $g$ are equivalent Thurston maps and one of them is expanding, then the other one is not necessarily expanding as well. Thus, topological conjugacy is a much stronger condition than Thurston equivalence. The following theorem (see Theorem 9.2 in [3]) shows that under the condition that both maps are expanding, these two relations are the same.

Theorem II.4. Let $f: \mathbb{S}^{2} \rightarrow \mathbb{S}^{2}$ and $g: \mathbb{S}_{1}^{2} \rightarrow \mathbb{S}_{1}^{2}$ be equivalent Thurston maps that are expanding. Then they are topologically conjugate.

We now consider the cardinality of the postcritical set of $f$. In Remark 5.5 in [3], it is proved that there are no Thurston maps with \# post $(f) \leq 1$. Proposition 6.2 in [3] shows that all Thurston maps with \# post $(f)=2$ are Thurston equivalent to a power map on the Riemann sphere,

$$
z \mapsto z^{k} \text {, for some } k \in \mathbb{Z} \backslash\{-1,0,1\} \text {. }
$$

Corollary 6.3 in [3] states that if $f: \mathbb{S}^{2} \rightarrow \mathbb{S}^{2}$ is an expanding Thurston map, then $\# \operatorname{post}(f) \geq 3$. 
Let $f: \mathbb{S}^{2} \rightarrow \mathbb{S}^{2}$ be a Thurston map, and let $\mathcal{C} \subseteq \mathbb{S}^{2}$ be a Jordan curve containing $\operatorname{post}(f)$. By the Schönflies theorem, the set $\mathbb{S}^{2} \backslash \mathcal{C}$ has two connected components, which are both homeomorphic to the open unit disk. Let $T_{0}$ and $T_{0}^{\prime}$ denote the closures of these components. They are cells of dimension 2, which we call 0-tiles. The postcritical points of $f$ are called 0-vertices of $T_{0}$ and $T_{0}^{\prime}$, singletons of which are cells of dimension 0 . We call the closed arcs between vertices 0 -edges of $T_{0}$ and $T_{0}^{\prime}$, which are cells of dimension 1 . These 0 -vertices, 0 -edges and 0 -tiles form a cell decomposition of $\mathbb{S}^{2}$, denoted by $\mathcal{D}^{0}=\mathcal{D}^{0}(f, \mathcal{C})$. We call the elements in $\mathcal{D}^{0} 0$-cells. Let $\mathcal{D}^{1}=\mathcal{D}^{1}(f, \mathcal{C})$ be the set of connected subsets $c \subseteq \mathbb{S}^{2}$ such that $f(c)$ is a cell in $\mathcal{D}^{0}$ and $\left.f\right|_{c}$ is a homeomorphism of $c$ onto $f(c)$. Call $c$ a 1-tile if $f(c)$ is a 0 -tile, call $c$ a 1-edge if $f(c)$ is a 0 -edge, and call $c$ a 1 -vertex if $f(c)$ is a 1-vertex. Lemma 5.4 in [3] states that $\mathcal{D}^{1}$ is a cell decomposition of $\mathbb{S}^{2}$. Continuing in this manner, let $\mathcal{D}^{n}=\mathcal{D}^{n}(f, \mathcal{C})$ be the set of all connected subsets of $c \subseteq \mathbb{S}^{2}$ such that $f(c)$ is a cell in $\mathcal{D}^{n-1}$ and $\left.f\right|_{c}$ is a homeomorphism of $c$ onto $f(c)$, and call these connected subsets $n$-tiles, $n$-edges and $n$-vertices correspondingly, for $n \in \mathbb{N}_{0}$. By Lemma 5.4 in [3], $\mathcal{D}^{n}$ is a cell decomposition of $\mathbb{S}^{2}$, for each $n \in \mathbb{N}_{0}$, and we call the elements in $\mathcal{D}^{n} n$-cells. The following lemma lists some properties of these cell decompositions. For more details, we refer the reader to Proposition 6.1 in [3].

Lemma II.5. Let $k, n \in \mathbb{N}_{0}$, let $f: \mathbb{S}^{2} \rightarrow \mathbb{S}^{2}$ be a Thurston map, let $\mathcal{C} \subset \mathbb{S}^{2}$ be a Jordan curve with $\mathcal{C} \supseteq \operatorname{post}(f)$, and let $m=\# \operatorname{post}(f)$.

1. If $\tau$ is any $(n+k)$-cell, then $f^{k}(\tau)$ is an $n$-cell, and $\left.f^{k}\right|_{\tau}$ is a homeomorphism of $\tau$ onto $f^{k}(\tau)$.

2. Let $\sigma$ be an $n$-cell. Then $f^{-k}(\sigma)$ is equal to the union of all $(n+k)$-cells $\tau$ with $f^{k}(\tau)=\sigma$ 
3. The number of n-vertices is less than or equal to $m \operatorname{deg}(f)^{n}$, the number of $n$-edges is $m \operatorname{deg}(f)^{n}$, and the number of $n$-tiles is $2 \operatorname{deg}(f)^{n}$.

4. The $n$-edges are precisely the closures of the connected components of $f^{-n}(\mathcal{C}) \backslash$ $f^{-n}(\operatorname{post}(f))$. The $n$-tiles are precisely the closures of the connected components of $\mathbb{S}^{2} \backslash f^{-n}(\mathcal{C})$.

5. Every $n$-tile is an m-gon, i.e., the number of $n$-edges and n-vertices contained in its boundary is equal to $\mathrm{m}$.

Let $\sigma$ be an $n$-cell. Let $W^{n}(\sigma)$ be the union of the interiors of all $n$-cells intersecting with $\sigma$, and call $W^{n}(\sigma)$ the $n$-flower of $\sigma$. In general, $W^{n}(\sigma)$ is not necessarily simply connected. The following lemma (from Lemma 7.2 in [3]) says that if $\sigma$ consists of a single $n$-vertex, then $W^{n}(\sigma)$ is simply connected.

Lemma II.6. Let $f: \mathbb{S}^{2} \rightarrow \mathbb{S}^{2}$ be a Thurston map, and let $\mathcal{C}$ be a Jordan curve containing $\operatorname{post}(f)$. If $\sigma$ is an n-vertex, then $W^{n}(\sigma)$ is simply connected. In addition, the closure of $W^{n}(\sigma)$ is the union of all n-tiles containing the vertex $\sigma$.

One of the most important properties of $n$-flowers is that they build a connection between $n$-tiles of different Jordan curves due to the following lemma in [3, Lemma $7.12]$

Lemma II.7. Let $\mathcal{C}$ and $\mathcal{C}^{\prime}$ be Jordan curves in $\mathbb{S}^{2}$ both containing post $(f)$. Then there exists a number $M$ such that each $n$-tile for $(f, \mathcal{C})$ is covered by $M n$-flowers for $\left(f, \mathcal{C}^{\prime}\right)$.

Remark 1. The exact same proof for this lemma shows that for $n^{\prime} \geq n$, there exists a number $M$ such that each $n^{\prime}$-tile $(f, \mathcal{C})$ is covered by $M n$-flowers for $\left(f, \mathcal{C}^{\prime}\right)$.

We obtain a sequence of cell decompositions of $\mathbb{S}^{2}$ from a Thurston map and a Jordan curve on $\mathbb{S}^{2}$. It would be nice if the local degrees of the map $f$ at all the 
vertices were bounded, and this can be obtained by the assumption of no periodic critical points (see [3, Lemma 16.1]).

Lemma II.8. Let $f: \mathbb{S}^{2} \rightarrow \mathbb{S}^{2}$ be a branched covering map. Then $f$ has no periodic critical points if and only if there exists $N \in \mathbb{N}$ such that

$$
\operatorname{deg}_{f^{n}}(p) \leq N
$$

for all $p \in \mathbb{S}^{2}$ and all $n \in \mathbb{N}$.

Henceforth we assume that all Thurston maps have no periodic critical points.

Definition II.9. Let $f: \mathbb{S}^{2} \rightarrow \mathbb{S}^{2}$ be an expanding Thurston map, and let $\mathcal{C} \subseteq \mathbb{S}^{2}$ be a Jordan curve containing post $(f)$. Let $x, y \in \mathbb{S}^{2}$. For $x \neq y$ we define

$$
\begin{array}{r}
m_{f, \mathcal{C}}(x, y)=\min \left\{n \in \mathbb{N}_{0}: \text { there exist disjoint } n \text {-tiles } X \text { and } Y\right. \\
\text { for }(f, \mathcal{C}) \text { with } x \in X \text { and } y \in Y\} .
\end{array}
$$

If $x=y$, we define $m_{f, \mathcal{C}}(x, x)=\infty$.

The minimum in the definition above is always obtained since the diameters of $n$-tiles go to 0 as $n \rightarrow \infty$. We usually drop one or both subscripts in $m_{f, \mathcal{C}}(x, y)$ if $f$ or $\mathcal{C}$ is clear from the context. If we define for $x, y \in \mathbb{S}^{2}$ and $x \neq y$,

$$
\begin{array}{r}
m_{f, \mathcal{C}}^{\prime}(x, y)=\max \left\{n \in \mathbb{N}_{0}: \text { there exist nondisjoint } n \text {-tiles } X \text { and } Y\right. \\
\text { for }(f, \mathcal{C}) \text { with } x \in X \text { and } y \in Y\},
\end{array}
$$

then $m_{f, \mathcal{C}}$ and $m_{f, \mathcal{C}}^{\prime}$ are essentially the same up to a constant (see Lemma 8.6 (v) in $[3])$.

Lemma II.10. Let $m_{f, \mathcal{C}}$ and $m_{f, \mathcal{C}}^{\prime}$ as defined above. There exists a constant $k>0$, such that for any $x, y \in \mathbb{S}^{2}$ and $x \neq y$,

$$
m_{f, \mathcal{C}}(x, y)-k \leq m_{f, \mathcal{C}}^{\prime}(x, y) \leq m_{f, \mathcal{C}}(x, y)+1
$$


Definition II.11. Let $f: \mathbb{S}^{2} \rightarrow \mathbb{S}^{2}$ be an expanding Thurston map and $d$ be a metric on $\mathbb{S}^{2}$. The metric $d$ is called a visual metric for $f$ if there exists a Jordan curve $\mathcal{C} \subseteq \mathbb{S}^{2}$ containing $\operatorname{post}(f)$, constants $\Lambda>1$ and $C \geq 1$ such that

$$
\frac{1}{C} \Lambda^{-m_{f, \mathcal{C}}(x, y)} \leq d(x, y) \leq C \Lambda^{-m_{f, \mathcal{C}}(x, y)}
$$

for all $x, y \in \mathbb{S}^{2}$.

Proposition 8.9 in [3] states that for any expanding Thurston map $f: \mathbb{S}^{2} \rightarrow \mathbb{S}^{2}$, there exists a visual metric for $f$, which induces the standard topology on $\mathbb{S}^{2}$. Lemma 8.10 in the same paper gives the following characterization of visual metrics.

Lemma II.12. Let $f: \mathbb{S}^{2} \rightarrow \mathbb{S}^{2}$ be an expanding Thurston map. Let $\mathcal{C} \subseteq \mathbb{S}^{2}$ be a Jordan curve containing post $(f)$, and $d$ be a visual metric for $f$ with expansion factor $\Lambda>1$. Then there exists a constant $C>1$ such that

1. $d(\sigma, \tau) \geq(1 / C) \Lambda^{-n}$ whenever $\sigma$ and $\tau$ are disjoint $n$-cells,

2. $(1 / C) \Lambda^{-n} \leq \operatorname{diam}(\tau) \leq C \Lambda^{-n}$ for $\tau$ as any $n$-edge or $n$-tile.

Conversely, if $d$ is a metric on $\mathbb{S}^{2}$ satisfying conditions (1) and (2) for some constant $C>1$, then $d$ is a visual metric with expansion factor $\Lambda>1$.

Let $(X, d)$ be a metric space. For $\alpha \geq 0$ and for any Borel subset $S \subseteq X$, the $\alpha$-dimensional Hausdorff measure $H^{\alpha}(S)$ of $S$ is defined as

$$
H^{\alpha}(S):=\lim _{\epsilon \rightarrow 0} H_{\epsilon}^{\alpha}(S)
$$

where

$$
H_{\epsilon}^{\alpha}(S)=\inf \left\{\sum_{i=1}^{\infty} \operatorname{diam}\left(U_{i}\right)^{\alpha}: S \subseteq \bigcup_{i=1}^{\infty} U_{i} \text { and } \operatorname{diam}\left(U_{i}\right)<\epsilon\right\}
$$

where the infimum is taking over all countable covers $\left\{U_{i}\right\}$ of $S$. The Hausdorff dimension $\operatorname{dim}_{H}(X)$ of a metric space $X$ is the infimum of the set of $\alpha \in[0, \infty)$ such 
that $\alpha$-dimensional Hausdorff measure of $X$ is zero:

$$
\operatorname{dim}_{H}(X):=\inf \left\{\alpha \geq 0: H^{\alpha}(X)=0\right\}
$$

The dimension of a probability measure $\mu$ on $X$ is

$$
\operatorname{dim} \mu:=\inf \left\{\operatorname{dim}_{H}(E): E \subset X \text { is measurable and } \mu(E)=1\right\} .
$$

The following theorem ([17, Theorem 4]) gives a characterization of Lattès maps among among all expanding rational Thurston maps.

Theorem II.13. Let $f: \widehat{\mathbb{C}} \rightarrow \widehat{\mathbb{C}}$ be an expanding rational Thurston map. The map $f$ is a Lattès map if and only if there exists a visual metric $d$ on $\widehat{\mathbb{C}}$ such that the dimension of the (normalized standard) Lebesgue measure with respect to the metric $d$ is equal to 2 .

\subsection{Gromov Hyperbolic Spaces}

Let us first review some basic facts about Gromov hyperbolic spaces. We refer the reader to [5] as a general source on Gromov hyperbolic spaces. Let $(X, d)$ be a geodesic metric space. For any points $x, y, p \in X$, the Gromov product $(x, y)_{p}$ of $x$ and $y$ with respect to base point $p$ is defined as

$$
(x, y)_{p}:=\frac{1}{2}[d(x, p)+d(y, p)-d(x, y)]
$$

The space $X$ is called $\delta$-hyperbolic (or Gromov hyperbolic) for some $\delta \geq 0$ if there exists a base point $p \in X$, such that for all $x, y, z \in X$ we have

$$
(x, y)_{p} \geq \min \left\{(x, z)_{p},(z, y)_{p}\right\}-\delta
$$

If this inequality holds for some base point $p \in X$, then it also holds for any other $p^{\prime} \in X$ with $\delta$ being replaced by $2 \delta$. 
Let $(X, d)$ be a Gromov hyperbolic metric space with a fixed base point $p \in X$. A sequence of points $\left\{x_{i}\right\} \subseteq X$ converges to infinity if

$$
\lim _{i, j \rightarrow \infty}\left(x_{i}, x_{j}\right)_{p}=\infty
$$

This property of a sequence $\left\{x_{i}\right\}$ does not depend on the base point $p \in X$. We say two sequences converging to infinity $\left\{x_{i}\right\}$ and $\left\{x_{i}^{\prime}\right\}$ are equivalent if

$$
\lim _{i \rightarrow \infty}\left(x_{i}, x_{i}^{\prime}\right)_{p}=\infty
$$

The boundary at infinity $\partial_{\infty} X$ of $X$ is defined to be the set of equivalence classes of sequences of points converging to infinity. One can also define the Gromov product for points $\xi, \xi^{\prime} \in \partial_{\infty} X$ and $p \in X$ as

$$
\left(\xi, \xi^{\prime}\right)_{p}:=\inf \liminf _{i \rightarrow \infty}\left(x_{i}, x_{i}^{\prime}\right)_{p}
$$

where the infimum is taken over all sequences $\left\{x_{i}\right\} \in \xi$ and $\left\{x_{i}^{\prime}\right\} \in \xi^{\prime}$. Here $\left(\xi, \xi^{\prime}\right)_{p}=$ $\infty$ if and only if $\xi=\xi^{\prime}$.

A metric $\rho$ on the boundary at infinity $\partial_{\infty} X$ of a Gromov hyperbolic space $X$ is called visual if there exist $p \in X, \Lambda>1$ and $k \geq 1$ such that for all $\xi, \xi^{\prime} \in \partial_{\infty} X$, we have that

$$
\frac{1}{k} \Lambda^{-\left(\xi, \xi^{\prime}\right)_{p}} \leq \rho\left(\xi, \xi^{\prime}\right) \leq k \Lambda^{-\left(\xi, \xi^{\prime}\right)_{p}}
$$

We call the constant $\Lambda$ in this inequality the expansion factor of the visual metric $\rho$. Recall that we also defined a visual metric for an expanding Thurston map (see Definition 2.11). When it is not clear from context, we will refer to the visual metric defined in (2.4) as a 'visual metric in the Gromov hyperbolic sense'.

Given two metric spaces $\left(X, d_{X}\right)$ and $\left(Y, d_{Y}\right)$, a map $f: X \rightarrow Y$ is called a quasiisometry if there are constants $\lambda \geq 1$ and $k \geq 0$ such that for all $x, x^{\prime} \in X$

$$
\frac{1}{\lambda} d_{X}\left(x, x^{\prime}\right)-k \leq d_{Y}\left(f(x), f\left(x^{\prime}\right)\right) \leq \lambda d_{X}\left(x, x^{\prime}\right)+k
$$


and for all $y \in Y$,

$$
\inf _{x \in X} d_{Y}(f(x), y) \leq k
$$

If $\lambda=1$, we call the map $f$ a rough-isometry. We say that the spaces $X$ and $Y$ are quasi-isometric (rough-isometric) if there is a quasi-isometry (rough-isometry) between them.

In [1], Bonk and Foertsch introduced the notion of upper curvature bounds for Gromov hyperbolic spaces up to rough-isometry (see [1, Definition 1.1 and 1.2]).

Definition II.14. Let $\kappa \in[-\infty, 0)$. We call a metric space $X$ an $A C_{u}(\kappa)$-space if there exists $p \in X$ and a constant $c \geq 0$ such that for all $x, x^{\prime} \in X$ and all finite sequences $x_{0}=x, x_{1}, \ldots, x_{n}=x^{\prime}$ in $X$ with $n>0$,

$$
\left(x, x^{\prime}\right)_{p} \geq \min _{i=1, \ldots, n}\left(x_{i-1}, x_{i}\right)_{p}-\frac{1}{\sqrt{-\kappa}} \log n-c .
$$

Here we use the convention $1 / \sqrt{\infty}=0$. We call

$$
K_{u}(X):=\inf \left\{\kappa: X \text { is an } \mathrm{AC}_{u}(\kappa) \text {-space } \in[-\infty, 0)\right\}
$$

the asymptotic upper curvature of $X$.

Rough-isometric Gromov hyperbolic spaces have the same asymptotic upper curvature since under rough-isometries, Gromov products only change by a fixed additive amount, which can be absorbed in the constant $c$ in $(2.5)$.

The asymptotic upper curvature is related to the expansion factors of visual metrics in Gromov hyperbolic spaces, due to the following theorem [1, Theorem 1.5].

Theorem II.15. Let $X$ be a Gromov hyperbolic metric space. If there exists a visual metric on $\partial_{\infty} X$ with expansion factor $\Lambda>1$, then $X$ is an $A C_{u}(\kappa)$-space with $\kappa=-\log ^{2} \Lambda$. Conversely, if $X$ is an $A C_{u}(\kappa)$-space, then for every $1<\Lambda<e^{\sqrt{-\kappa}}$, 
there exists a visual metric on $\partial_{\infty} X$ with expansion $\Lambda$. In particular,

$$
K_{u}(X)=-\log ^{2} \Lambda_{0},
$$

where

$$
\begin{array}{r}
\Lambda_{0}:=\sup \left\{\Lambda: \quad \text { there exists a visual metric on } \partial_{\infty} X\right. \\
\text { with expansion factor } \Lambda\} .
\end{array}
$$




\section{CHAPTER III}

\section{Lattès Maps and Combinatorial Expansion}

\subsection{Lattès and Lattès-type Maps}

In this section, we introduce Lattès-type maps and establish some of their properties. We also briefly review the concept of the orbifold $O_{f}$ of a Thurston map $f$.

Let $\Lambda, \Lambda^{\prime} \subseteq \mathbb{R}^{2}$ be lattices (we always assume that a lattice has rank 2). The quotients $\mathcal{T}=\mathbb{R}^{2} / \Lambda$ and $\mathcal{T}^{\prime}=\mathbb{R}^{2} / \Lambda^{\prime}$ are tori. Let $A: \mathbb{R}^{2} \rightarrow \mathbb{R}^{2}$ be an affine orientation-preserving map such that for any two points $p, q \in \mathbb{R}^{2}$ with $p-q \in \Lambda$, we have $A(p)-A(q) \in \Lambda^{\prime}$. The quotient of map $A$,

$$
\bar{A}: \mathcal{T} \rightarrow \mathcal{T}^{\prime}
$$

is called an (orientation-preserving) torus homomorphism. If the map $\bar{A}$ is also bijective, we call the map $\bar{A}: \mathcal{T} \rightarrow \mathcal{T}^{\prime}$ a torus isomorphism between $\mathcal{T}$ and $\mathcal{T}^{\prime}$. If $\Lambda=\Lambda^{\prime}$, we call the induced map $\bar{A}: \mathcal{T} \rightarrow \mathcal{T}$ a torus endomorphism. If in addition, the map $\bar{A}$ is a torus isomorphism, then we call $\bar{A}$ a torus automorphism of $\mathcal{T}$. An affine map $A$ which induces a torus endomorphism has the form

$$
A\left(\begin{array}{l}
x \\
y
\end{array}\right)=L\left(\begin{array}{l}
x \\
y
\end{array}\right)+\left(\begin{array}{l}
x_{0} \\
y_{0}
\end{array}\right) \text { for any }\left(\begin{array}{l}
x \\
y
\end{array}\right) \in \mathbb{R}^{2},
$$

where $L$ is a $2 \times 2$ matrix with integer entries and positive determinant, and $x_{0}, y_{0} \in \mathbb{R}$. If $A$ induces a torus automorphism, then $L \in \mathrm{SL}(2, \mathbb{Z})$. 
The matrix $L$ is uniquely determined by $\bar{A}$. Indeed, if affine maps $A$ and $A^{\prime}$ induce the same torus endomorphism, then $A$ and $A^{\prime}$ differ by a translation by $\lambda$ according to equation (3.1), where $\lambda \in \Lambda$. Define the determinant, trace and eigenvalues of a torus endomorphism $\bar{A}$ and the determinant, trace and eigenvalues of the affine map $A$ to be the determinant, trace and eigenvalues of the matrix $L$ as in equation (3.1) respectively, which are uniquely determined. Denote

$$
\operatorname{det} \bar{A}=\operatorname{det} A=\operatorname{det} L, \quad \operatorname{tr}(\bar{A})=\operatorname{tr}(A)=\operatorname{tr}(L)
$$

If $\bar{A}$ is a torus endomorphism, we refer to the corresponding $\mathbb{R}$-linear map $A: \mathbb{R}^{2} \rightarrow$ $\mathbb{R}^{2}$ as the lift of $\bar{A}$.

Definition III.1. We call $\Theta: \mathcal{T} \rightarrow \mathbb{S}^{2}$ a branched cover induced by a rigid action of a group $G$ on $\mathcal{T}$ if every element of $g \in G$ acts as a torus automorphism and for any $t, t^{\prime} \in \mathcal{T}$, we have $\Theta(t)=\Theta\left(t^{\prime}\right)$ if and only if there exists $g \in G$ such that

$$
t=g\left(t^{\prime}\right)
$$

An equivalent formulation is that $\Theta$ induces a canonical homeomorphism from the quotient space $\mathcal{T} / G$ onto $\mathbb{S}^{2}$.

Definition III.2. Let $\Lambda \subseteq \mathbb{R}^{2}$ be a lattice. Let $\bar{A}$ be a torus endomorphism of $\mathcal{T}=$ $\mathbb{R}^{2} / \Lambda$ whose both eigenvalues have absolute values greater than 1 . Let $\Theta: \mathcal{T} \rightarrow \mathbb{S}^{2}$ be a branched covering map induced by a rigid action of a finite cyclic group on $\mathcal{T}$. A map $f: \mathbb{S}^{2} \rightarrow \mathbb{S}^{2}$ is called a Lattès-type map (with respect to a lattice $\Lambda$ ) if there exists $\bar{A}$ as above such that the semi-conjugacy relation $f \circ \Theta=\Theta \circ \bar{A}$ is satisfied, i.e., the following diagram commutes:

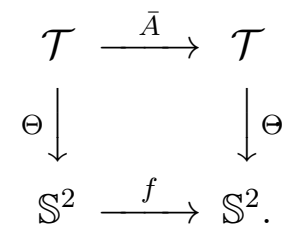


In addition, if a Lattès-type map $f$ is rational, then the map $f$ is called a Lattès map.

We remark that this definition of Lattès maps is equivalent to the definition of Lattès maps in [18].

Lemma III.3. A Lattès-type map $f$ over any lattice $\Lambda$ is a Lattès-type map over the integer lattice $\mathbb{Z}^{2}$.

Proof. For any Lattès-type map $f$ over a lattice $\Lambda$, let $\mathcal{T}=\mathbb{R}^{2} / \Lambda$. There exist a torus endomorphism

$$
\bar{A}: \mathcal{T} \rightarrow \mathcal{T}
$$

and a branched covering map $\Theta: \mathcal{T} \rightarrow \mathbb{S}^{2}$ induced by a rigid action of a fixed cyclic group on $\mathcal{T}$, such that $f \circ \Theta=\Theta \circ \bar{A}$. Let $\mathcal{T}_{0}=\mathbb{R}^{2} / \mathbb{Z}^{2}$. Since $\Lambda$ is a lattice with rank 2 , there is an orientation-preserving isomorphism $L: \mathbb{Z}^{2} \rightarrow \Lambda$. This isomorphism $L$ can be extended to an $R$-linear map of $\mathbb{R}^{2}$, still denoted it by $L$, which induces a torus isomorphism $L: \mathcal{T}_{0} \rightarrow \mathcal{T}$. Define a map

$$
A_{0}: \mathcal{T}_{0} \rightarrow \mathcal{T}_{0}
$$

by $A_{0}=L^{-1} \circ \bar{A} \circ L$, and a branched covering map $\Theta_{0}: \mathcal{T}_{0} \rightarrow \mathbb{S}^{2}$ by $\Theta_{0}=\Theta \circ L$. Hence, $A_{0}$ is a torus endomorphism and the branched covering map $\Theta_{0}$ is induced by a rigid action of a finite cyclic group on $\mathcal{T}_{0}$. In addition,

$$
f \circ \Theta_{0}=f \circ \Theta \circ L=\Theta \circ \bar{A} \circ L=(\Theta \circ L) \circ\left(L^{-1} \circ \bar{A} \circ L\right)=\Theta_{0} \circ A_{0} .
$$

See the diagram below. It follows that the map $f$ is a Lattès-type map over the 
lattice $\mathbb{Z}^{2}$.

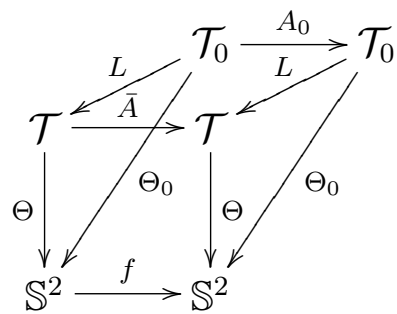

Remark 2. Notice that the proof of this lemma works for any rank-2 lattice besides the integer lattice $\mathbb{Z}^{2}$. Hence, we can choose the lattice for our convenience.

Lemma III.4. If a branched covering map $\Theta: \mathcal{T} \rightarrow \mathbb{S}^{2}$ is induced by a rigid action of a finite cyclic group $G$ on $\mathcal{T}$, then the order of $G$ is necessarily 2,3, 4 or 6 .

Proof. Let $g$ be a generator of $G$. The order of $g$ is greater than 1 since

$$
\mathcal{T} / G=\mathbb{S}^{2}
$$

The element $g$ is an automorphism of the torus $\mathcal{T}$, so $g$ is induced by an affine map $A_{g}$ of $\mathbb{R}^{2}$, i.e., $A_{g}$ has a form

$$
A_{g}\left(\begin{array}{l}
x \\
y
\end{array}\right)=L_{g}\left(\begin{array}{l}
x \\
y
\end{array}\right)+\left(\begin{array}{l}
x_{g} \\
y_{g}
\end{array}\right) \text { for any }\left(\begin{array}{l}
x \\
y
\end{array}\right) \in \mathbb{R}^{2},
$$

where $L_{g} \in \mathrm{SL}(2, \mathbb{Z})$ and $x_{g}, y_{g} \in \mathbb{R}$. Since $L_{g} \in \mathrm{SL}(2, \mathbb{Z})$ and

$$
L_{g}^{n}=I,
$$

the matrix $L_{g}$ is conjugate to a rotation of $\mathbb{R}^{2}$,

$$
\left(\begin{array}{cc}
\cos \frac{2 \pi}{n} & \sin \frac{2 \pi}{n} \\
-\sin \frac{2 \pi}{n} & \cos \frac{2 \pi}{n}
\end{array}\right)
$$

In addition, since the trace of $L_{g}$ is an integer, we must have

$$
2 \cos \frac{2 \pi}{n} \in \mathbb{Z}
$$


Hence, the order $n$ of the group $G$ can only be $2,3,4$ or 6 .

Proposition III.5. Every Lattès-type map $f$ is an expanding Thurston map.

Proof. By Lemma III.3, we know that $f$ is a Lattès-type map over the lattice $\mathbb{Z}^{2}$. Let $\mathcal{T}=\mathbb{R}^{2} / \mathbb{Z}^{2}$. There exists a torus endomorphism

$$
\bar{A}: \mathcal{T} \rightarrow \mathcal{T}
$$

and a branched covering map $\Theta: \mathcal{T} \rightarrow \mathbb{S}^{2}$ induced by a rigid action of a finite cyclic group $G$ on $\mathcal{T}$, such that $f \circ \Theta=\Theta \circ \bar{A}$. Let $A: \mathbb{R}^{2} \rightarrow \mathbb{R}^{2}$ be an affine map inducing $\bar{A}$.

The map $\Theta \circ \bar{A}$ is a branched covering map since locally $\bar{A}$ is a homeomorphism and $\Theta$ can be written as $v \mapsto v^{d}$, where $v=\bar{A}(z)$ and $d=\operatorname{deg}_{\Theta \circ \bar{A}}(z)=\operatorname{deg}_{\Theta}(\bar{A}(z))$. Locally, the map $\Theta$ can be written as $z \mapsto z^{d^{\prime}}$, where $d^{\prime}=\operatorname{deg}_{\Theta}(z)$. Since $\bar{A}$ has local degree 1 on every point, we have

$$
\operatorname{deg}_{f}(\Theta(z)) \operatorname{deg}_{\Theta}(z)=\operatorname{deg}_{\Theta}(\bar{A}(z))
$$

Since $f \circ \Theta=\Theta \circ \bar{A}, f$ can be locally written as $w \mapsto w^{d / d^{\prime}}$, where $w=\Theta(z)$ and $d / d^{\prime}=\operatorname{deg}_{f}(w)$ is an integer. Hence, a Lattès-type map $f$ is a branched covering map.

Let $V_{f}$ and $V_{\Theta}$ be the sets of critical values of $f$ and $\Theta$ respectively. We claim that $\operatorname{post}(f)=V_{\Theta}$ and they have finite cardinality. The claim is proved similarly to Lemma 3.4 in [18]. We give the details of the argument for the convenience of the reader. Since $\bar{A}$ locally is a homeomorphism and $\bar{A}$ and $\Theta$ are both surjective, a point $p \in \mathbb{S}^{2}$ is a critical value of $\Theta$ if and only if either $p$ is a critical value of $f$, or $p$ has a preimage in $f^{-1}(p)$, which is a critical value of $\Theta$. So $V_{\Theta}=V_{f} \cup f\left(V_{\Theta}\right)$. Hence $f\left(V_{f}\right) \subseteq f\left(V_{\Theta}\right) \subseteq V_{\Theta}$ and inductively, we have $\operatorname{post}(f) \subseteq V_{\Theta}$. The set $V_{\Theta}$ is finite 
due to the compactness of $\mathbb{S}^{2}$, and so is the set of critical points of $\Theta$. Assume that there exists a critical point $t \in \mathcal{T}$ of $\Theta$ such that $\Theta(t)$ is not in post $(f)$. There exists $t_{1} \neq t$ in the preimage of $t$ under $\bar{A}$, and there exists $t_{2} \neq t_{1}, t$ in the preimage of $t_{1}$ under $\bar{A}$, and inductively the cardinality of $\left\{t_{i}\right\}$ is not finite. Each $t_{i}$ is a critical point of $\Theta$. This is a contradiction to the finiteness of the critical set of $\Theta$.

We claim that $\operatorname{deg}(f)=\operatorname{det}(A)$. Since $\mathcal{T}=\mathbb{R}^{2} / \mathbb{Z}^{2}$, $\operatorname{deg}(\bar{A})$ is equal to the minimum number of parallelograms $A^{-1}(I \times I)$ needed to cover $I \times I$, where $I=[0,1]$. By the area argument, we have

$$
\operatorname{deg}(\bar{A})=\frac{\operatorname{area}(I \times I)}{\operatorname{area}\left(A^{-1}(I \times I)\right)}=\frac{1}{\operatorname{det}\left(A^{-1}\right)}=\operatorname{det}(A)
$$

Notice that $\operatorname{deg}(f)=\operatorname{deg}(\bar{A})$. The claim follows. Since $\operatorname{deg}(f)=\operatorname{det}(A)>1$, the Lattès map $f$ is a Thurston map.

Let $\mathcal{C} \subseteq \mathbb{S}^{2}$ be a Jordan curve containing post $(f)$. The torus $\mathcal{T}$ carries a flat metric induced by the Euclidean metric $\mathbb{R}^{2}$. Since every element in $G$ acting on $\mathcal{T}$ is a torus automorphism, $\Theta$ induces a flat metric on $\mathcal{T} / G \cong \mathbb{S}^{2}$. Observe that the interior $T$ of an $n$-tile under the cell decomposition of $(f, \mathcal{C})$ does not intersect with $\operatorname{post}(f)=V_{\Theta}$, so $\Theta$ restricted to one of the connected components $T^{\prime}$ of $\Theta^{-1}(T)$ is a homeomorphism, denoted by $\Theta_{T^{\prime}}$. In addition, the homeomorphism $\Theta_{T^{\prime}}$ is actually an isometry, which implies that

$$
\operatorname{diam}(T)=\operatorname{diam}\left(T^{\prime}\right)
$$

Each connected component of $\bar{A}^{-1}\left(T^{\prime}\right)$ has diameter $\lambda$ diam $\left(T^{\prime}\right)$, where

$$
\lambda=\left|\lambda_{1}\right|^{-1}<1
$$

is the inverse of the smaller absolute value of eigenvalues of $\bar{A}$. Hence, each connected component of preimage of an $n$-tile $T$ under $f$ has diameter $\lambda^{n} \operatorname{diam}(T)$, where $\lambda<1$. 
Therefore, we have

$$
\operatorname{mesh}(f, n, \mathcal{C})=\lambda^{n} \operatorname{mesh}(f, 1, \mathcal{C}) \rightarrow 0
$$

as $n \rightarrow \infty$ and the map $f$ is expanding.

For $a, b \in \mathbb{Z} \cup\{\infty\}$, we use the convention that $\infty$ is a multiple of any positive integer or itself. If $a$ is a multiple of $b$, we denote $b \mid a$.

Lemma III.6. For any Thurston map $f$, there exists a unique smallest function $\nu_{f}$ among functions $\nu: \mathbb{S}^{2} \rightarrow \mathbb{N} \cup\{\infty\}$ such that

$$
\nu(p) \operatorname{deg}_{f}(p) \mid \nu(f(p))
$$

for all $p \in \mathbb{S}^{2}$.

Proof. We set $\nu(p)=1$ if $p$ is not a postcritical point of $f$. We only need to assign a value to the finitely many postcritical points of $f$. If we let $\nu(p)=\infty$ when $p \in \operatorname{post}(f)$, this shows the existence of a such function $\nu$. We have a natural partial order

$$
\nu_{1} \leq \nu_{2} \text { if } \nu_{1}(p) \leq \nu_{2}(p)
$$

for all $p \in \mathbb{S}^{2}$. The existence of a smallest function follows from assigning values over a fixed finite set. Suppose that $\nu_{1} \neq \nu_{2}$ are both smallest functions satisfying condition (3.2). Let

$$
\nu_{3}(p):=\operatorname{gcd}\left\{\nu_{1}(p), \nu_{2}(p)\right\},
$$

where gcd stands for greatest common divisor. We claim that $\nu_{3}$ satisfies condition (3.2). Indeed,

$$
\operatorname{gcd}\left\{\nu_{1}(f(p)), \nu_{2}(f(p))\right\}=\nu_{3}(f(p))
$$


is a multiple of

$$
\begin{aligned}
\operatorname{gcd}\left\{\nu_{1}(p) \operatorname{deg}_{f}(p), \nu_{2}(p) \operatorname{deg}_{f}(p)\right\} & =\operatorname{gcd}\left\{\nu_{1}(p), \nu_{2}(p)\right\} \operatorname{deg}_{f}(p) \\
& =\nu_{3}(p) \operatorname{deg}_{f}(p) .
\end{aligned}
$$

Hence, we have $\nu_{3}<\nu_{1}, \nu_{2}$ which is a contradiction. We conclude that there exists a unique smallest function $\nu_{f}$ satisfying condition (3.2).

Thurston associated an orbifold $O_{f}=\left(\mathbb{S}^{2}, \nu_{f}\right)$ to a Thurston map $f$ through the smallest $\nu_{f}$ function in Lemma III.6 (see [7]). More precisely, for each $p \in \mathbb{S}^{2}$ with $v_{f}(p) \neq 1$, the point $p$ is a cone point with cone angle $2 \pi / v_{f}(p)$. For $\operatorname{post}(f)=$ $\left\{p_{1}, \ldots, p_{m}\right\}$, use $\left(\nu\left(p_{1}\right), \ldots, \nu\left(p_{m}\right)\right)$ to denote the type of $O_{f}$. We are not going into the geometric significance of the orbifold here, and we refer the reader to Chapter 13 in [22] for more details.

Definition III.7. For any Thurston map $f$ and the smallest function $\nu_{f}: \mathbb{S}^{2} \rightarrow$ $\mathbb{N} \cup\{\infty\}$ associated to $f$ satisfying condition (3.2), let

$$
\chi\left(O_{f}\right)=2-\sum_{p \in \operatorname{post}(f)}\left(1-\frac{1}{\nu_{f}(p)}\right) .
$$

- If $\chi\left(O_{f}\right)=0$, we say that the orbifold $O_{f}$ is parabolic;

- If $\chi\left(O_{f}\right)<0$, we say that the orbifold $O_{f}$ is hyperbolic.

We call $\chi\left(O_{f}\right)$ the Euler characteristic of the orbifold $O_{f}$ associated to $f$.

Remark 3. By Proposition 9.1 (i) in [7], $\chi\left(O_{f}\right) \leq 0$.

Lemma III.8. For a Lattès-type map $f$, the orbifold $O_{f}$ is parabolic. In particular, the number of cone points must be either three or four. Hence, the cardinality of the postcritical set of $f$ is either three or four. 
Proof. There exist a torus endomorphism $\bar{A}: \mathcal{T} \rightarrow \mathcal{T}$ and a branched covering map $\Theta: \mathcal{T} \rightarrow \mathbb{S}^{2}$ induced by a group action on $\mathcal{T}$ as a rotation around some base point in $\mathcal{T}$, such that $f \circ \Theta=\Theta \circ \bar{A}$. For any points $t_{1} \in \bar{A}^{-1}\left(t_{0}\right), t_{i} \in \mathcal{T}$, and $p_{1} \in f^{-1}\left(p_{0}\right)$, $p_{i} \in \mathbb{S}^{2}$ such that $\Theta\left(t_{i}\right)=p_{i}, i=0,1$, we have that

$$
\operatorname{deg}_{\Theta}\left(t_{0}\right)=\operatorname{deg}_{f}\left(p_{1}\right) \operatorname{deg}_{\Theta}\left(t_{1}\right)
$$

Define $\nu(\Theta(t))=\operatorname{deg}_{\Theta}(t)$, and $\nu(p)=1$ if $p \notin \operatorname{post}(f)$. Since $\Theta$ is induced by a group action, different preimages of $\Theta(t)$ under $\Theta$ all have the same degree. In the proof of Proposition III.5, we showed that post $(f)$ is equal to the set of critical values of $\Theta$, so $\nu$ is well-defined on $\mathbb{S}^{2}$. In addition,

$$
\nu\left(p_{0}\right)=\nu\left(f\left(p_{1}\right)\right)=\operatorname{deg}_{\Theta}\left(t_{0}\right)=\operatorname{deg}_{f}\left(p_{1}\right) \operatorname{deg}_{\Theta}\left(t_{1}\right)=\operatorname{deg}_{f}\left(p_{1}\right) \nu\left(p_{1}\right) .
$$

So $\nu$ is a function satisfying condition (3.2).

We claim that $\nu$ is the smallest function satisfying condition (3.2). Indeed, suppose that $\nu^{\prime}$ satisfying condition (3.2) is smaller than $\nu$. If $p_{1} \notin \operatorname{post}(f)$, then

$$
\nu^{\prime}\left(p_{0}\right)=\nu^{\prime}\left(f\left(p_{1}\right)\right)=\operatorname{deg}_{f}\left(p_{1}\right)=\nu\left(f\left(p_{1}\right)\right)=\nu\left(p_{0}\right)
$$

If $p_{1} \in \operatorname{post}(f)$, then there exists $n>0$ and $p \in f^{-n}\left(p_{1}\right)$, such that $p \notin \operatorname{post}(f)$. By induction on $n$, we get that $\nu^{\prime}\left(p_{1}\right)=\nu\left(p_{1}\right)$ and hence

$$
\nu^{\prime}\left(p_{0}\right)=\nu^{\prime}\left(f\left(p_{1}\right)\right)=\operatorname{deg}_{f}\left(p_{1}\right) \nu^{\prime}\left(p_{1}\right)=\operatorname{deg}_{f}\left(p_{1}\right) \nu\left(p_{1}\right)=\nu\left(f\left(p_{1}\right)\right)=\nu\left(p_{0}\right) .
$$

Thus, $\nu^{\prime}=\nu$ and our claim is proved.

By the proof of Proposition 9.1 (i) in [7], $\nu\left(f\left(p_{1}\right)\right)=\operatorname{deg}_{f}\left(p_{1}\right) \nu\left(p_{1}\right)$ implies that $f$ is a covering map of orbifolds $f: O_{f} \rightarrow O_{f}$, and again by Proposition 9.1 (ii), $\chi\left(O_{f}\right)=0$ and $O_{f}$ is parabolic. All the parabolic orbifolds are classified in Section 9 in [7], and they have type $(2,2,2,2),(3,3,3),(2,3,6)$ and $(2,4,4)$, and all have three or four cone points. 


\subsection{Combinatorial Expansion Factor and $D_{n}$}

In this section, we first review the definitions and some properties of the combinatorial expansion factor and $D_{n}$ of an expanding Thurston map. Then we prove a relation between $D_{n}$ and the operator norm of the torus map for Lattès-type maps, which gives the necessity of the third condition in Theorem III.41.

Let $f: \mathbb{S}^{2} \rightarrow \mathbb{S}^{2}$ be an expanding Thurston map and let $\mathcal{C}$ be a Jordan curve containing post $(f)$. First, we review some definitions and propositions from [3].

Definition III.9. A set $K \subseteq \mathbb{S}^{2}$ joins opposite sides of $\mathcal{C}$ if $\# \operatorname{post}(f) \geq 4$ and $K$ meets two disjoint 0-edges, or if $\# \operatorname{post}(f)=3$ and $K$ meets all three 0-edges.

Let $D_{n}=D_{n}(f, \mathcal{C})$ be the minimum number of $n$-tiles needed to join opposite sides of a Jordan curve $\mathcal{C}$. More precisely,

$$
\begin{array}{r}
D_{n}=\min \left\{N \in \mathbb{N} \text { : there exist } n \text {-tiles } X_{1}, \ldots, X_{N}\right. \text { such that } \\
\left.\bigcup_{j=1}^{N} X_{j} \text { is connected and joins opposite sides of } \mathcal{C}\right\} .
\end{array}
$$

Of course, $D_{n}$ depends on $f$ and $\mathcal{C}$.

We are going to need Lemma 7.9 in [3] in Section 3.4. It states that:

Lemma III.10. Let $n \in \mathbb{N}_{0}$, and let $K \subset \mathbb{S}^{2}$ be a connected set. If there exist two disjoint $n$-cells $\sigma$ and $\tau$ with $K \cap \sigma \neq \emptyset$ and $K \cap \tau \neq \emptyset$, then $f^{n}(K)$ joins opposite sides of $\mathcal{C}$.

Lemma 7.10 in [3] states that:

Lemma III.11. For $n, k \in \mathbb{N}_{0}$, every set of $(n+k)$-tiles whose union is connected and meets two disjoint $n$-cells contains at least $D_{k}$ elements.

Proposition 17.1 in [3] says that: 
Proposition III.12. For an expanding Thurston map $f: \mathbb{S}^{2} \rightarrow \mathbb{S}^{2}$, and a Jordan curve $\mathcal{C}$ containing post $(f)$, the limit

$$
\Lambda_{0}=\Lambda_{0}(f):=\lim _{n \rightarrow \infty} D_{n}(f, \mathcal{C})^{1 / n}
$$

exists and is independent of $\mathcal{C}$.

We call $\Lambda_{0}(f)$ the combinatorial expansion factor of $f$.

Proposition 17.2 in [3] states that:

Proposition III.13. If $f: \mathbb{S}^{2} \rightarrow \mathbb{S}^{2}$ and $g: \mathbb{S}_{1}^{2} \rightarrow \mathbb{S}_{1}^{2}$ are expanding Thurston maps that are topologically conjugate, then $\Lambda_{0}(f)=\Lambda_{0}(g)$.

Let $f$ be an expanding Thurston map. For any two Jordan curves $\mathcal{C}, \mathcal{C}^{\prime}$ with $\operatorname{post}(f) \subset \mathcal{C}, \mathcal{C}^{\prime}$, inequality $(17.1)$ in $[3]$ states that there exists a constant $c>0$ such that for all $n>0$,

$$
\frac{1}{c} D_{n}(f, \mathcal{C}) \leq D_{n}\left(f, \mathcal{C}^{\prime}\right) \leq c D_{n}(f, \mathcal{C})
$$

We obtain the following lemma:

Lemma III.14. With the notation above, $D_{n}(f, \mathcal{C}) \geq c(\operatorname{deg} f)^{n / 2}$ for some $c>0$ if and only if $D_{n}\left(f, \mathcal{C}^{\prime}\right) \geq c^{\prime}(\operatorname{deg} f)^{n / 2}$ for some constant $c^{\prime}>0$.

So we may say that $D_{n} \geq c(\operatorname{deg} f)^{n / 2}$ for the same constant $c>0$ without specifying Jordan curves.

Lemma III.15. Let $f$ and $g$ be two expanding Thurston maps that are topologically conjugate by a homeomorphism $h$. Let $\mathcal{C}$ be a Jordan curve on $\mathbb{S}^{2}$ containing post $(f)$, and $\mathcal{C}^{\prime}$ be the image of $\mathcal{C}$ under $h$. Then

$$
D_{n}(f, \mathcal{C})=D_{n}\left(g, \mathcal{C}^{\prime}\right)
$$

for all $n \geq 0$. 
This lemma follows directly from the definitions of $D_{n}$ and topological conjugacy.

Recall that the maximum norm (or $l^{\infty}$ norm) of a vector $v=\left(x_{1}, \ldots, x_{n}\right) \in \mathbb{R}^{n}$ is

$$
\|v\|_{\infty}=\max \left\{\left|x_{1}\right|, \ldots,\left|x_{n}\right|\right\}
$$

Let $A: \mathbb{R}^{n} \rightarrow \mathbb{R}^{n}$ be an $\mathbb{R}$-linear map. Then $\left(l^{\infty}\right)$ operator norm is

$$
\|A\|_{\infty}:=\max \left\{\|A v\|_{\infty}: v \in \mathbb{R}^{2},\|v\|_{\infty}=1\right\}
$$

Let $f$ be Lattès-type map over a lattice $\Lambda$ with orbifold type $(2,2,2,2)$. There exist a torus endomorphism $\bar{A}: \mathcal{T} \rightarrow \mathcal{T}$ and a branched covering map $\Theta: \mathcal{T} \rightarrow \mathbb{S}^{2}$ induced by a group action on $\mathcal{T}$, such that $f \circ \Theta=\Theta \circ \bar{A}$, where $\mathcal{T}=\mathbb{R}^{2} / \Lambda$. We use $A$ to denote the linear map lifted to the covering of $\mathcal{T}$. By the remark after Lemma III.3, we may assume $\Lambda=2 \mathbb{Z}^{2}$. For a Lattès-type map with orbifold type $(2,2,2,2)$, we can identify $\Theta \circ p: \mathbb{R}^{2} \rightarrow \mathbb{S}^{2}$, where $p: \mathbb{R}^{2} \rightarrow \mathbb{R}^{2} /\left(2 \mathbb{Z}^{2}\right)$ is the quotient map, with the Weierstrass function $\wp: \mathbb{R}^{2} \rightarrow \mathbb{S}^{2}$ with the lattice $2 \mathbb{Z}^{2}$. See the diagram below.

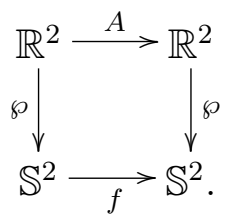

Let the Jordan curve $\mathcal{C}$ on $\mathbb{S}^{2}$ be the image of the boundary of the unit square $[0,1] \times[0,1]$ under $\wp$.

Proposition III.16. Let $f$ be a Lattès-type map with orbifold type $(2,2,2,2)$. Let $A$ be its corresponding linear map from $\mathbb{R}^{2}$ to $\mathbb{R}^{2}$ and $\wp: \mathbb{R}^{2} \rightarrow \mathbb{S}^{2}$ be the Weierstrass function with the lattice $2 \mathbb{Z}^{2}$ (as in the remark above). We have

$$
\frac{1}{\left\|A^{-n}\right\|_{\infty}} \leq D_{n}(f, \mathcal{C}) \leq \frac{1}{\left\|A^{-n}\right\|_{\infty}}+1
$$


where the Jordan curve $\mathcal{C}$ the image of the boundary of the unit square $[0,1] \times[0,1]$ under $\wp$.

Proof. Notice that the pre-image of $\mathcal{C}$ under $\wp$ is the whole grid of $\mathbb{Z}^{2}$ (i.e., the union of all the horizontal and vertical lines containing an integer valued point), and $\mathcal{C}$ contains all the post-critical points of $f$. The restriction of $\wp$ to the interior of the rectangle $R_{0}:=[0,2] \times[0,1]$ is a homeomorphism onto its image, which is the union of the interiors of the 0-tiles of $\mathbb{S}^{2}$ and one edge of a 0 -tile. Notice that the same holds for any rectangle obtained from two adjacent unit squares. The pre-images of unit squares under $A^{n}$ are parallelograms, which we call $n$-parallelograms. The $n$-tiles of $(f, \mathcal{C})$ (i.e., the pre-images of 0 -tiles under $\left.f^{n}\right)$ are the images of $n$-parallelograms under $\wp$. Let $D_{v}$ be the minimum number of $n$-parallelograms connecting the line $\{0\} \times(-\infty,+\infty)$ and $\{1\} \times(-\infty,+\infty)$, and let $D_{h}$ be the minimum number of $n$-parallelograms connecting the lines $(-\infty,+\infty) \times\{0\}$ and $(-\infty,+\infty) \times\{1\}$. We define

$$
D_{n}^{\prime}:=\min \left\{D_{v}, D_{h}\right\}
$$

We claim that $D_{n}=D_{n}^{\prime}$. Let $T_{1}, T_{2}, \ldots, T_{D_{n}}$ be a sequence with minimum numbers of $n$-tiles joining opposite sides of a 0 -tile. Without loss of generality, we may assume that this 0 -tile is the image of $[0,1] \times[0,1]$ under $\wp$, and the opposite sides of the 0 -tile are the images of the sides $[0,1] \times\{0\}$ and $[0,1] \times\{1\}$. Let $T_{1}^{\prime}=\wp^{-1}\left(T_{1}\right) \cap[0,1] \times[0,1]$, which is an $n$-parallelogram. Let $T_{2}^{\prime}$ be the path component of $\wp^{-1}\left(T_{2}\right)$ intersecting with $T_{1}^{\prime}$, which is also an $n$-parallelogram. Let $T_{3}^{\prime}$ be the path component of $\wp^{-1}\left(T_{3}\right)$ intersecting with $T_{2}^{\prime}$, and so on. We obtain a sequence of $n$-parallelograms $T_{1}^{\prime}, \ldots, T_{D_{n}}^{\prime}$ connecting $(-\infty,+\infty) \times\{0\}$ and $(-\infty,+\infty) \times\{1\}$, and hence $D_{n}^{\prime} \leq D_{n}$. On the other hand, given a sequence of $n$-parallelograms $P_{1}, \ldots, P_{m}$ connecting $(-\infty,+\infty) \times\{0\}$ and $(-\infty,+\infty) \times\{1\}$, or connecting $\{0\} \times(-\infty,+\infty)$ 
and $\{1\} \times(-\infty,+\infty)$, then $\wp\left(P_{1}\right), \ldots, \wp\left(P_{m}\right)$ is a sequence of $n$-tiles connecting a pair of opposite sides of a 0-tile. We conclude that $D_{n}^{\prime}=D_{n}$ as desired.

We next calculate $D_{n}^{\prime}$. Without loss of generality, we may assume that $D_{h} \leq D_{v}$, so that $D_{n}^{\prime}=D_{h}$. Observe that we need at least $m$ parallelograms to connect a pair of opposite sides of an $(m \times m)$-grid of parallelograms. Observe that

$$
A^{-n}([-m, m] \times[-m, m]) \cap(-\infty,+\infty) \times\{1\} \neq \emptyset
$$

if and only if there exist $m n$-parallelograms connecting $(-\infty,+\infty) \times\{0\}$ and $(-\infty,+\infty) \times\{1\}$. Hence, $D_{n}^{\prime}$ is equal to the smallest positive integer $m$ such that $y_{0}=\max \left\{y_{( \pm m, \pm m)}\right\}$ is greater than 1 , where

$$
\left(\begin{array}{l}
x_{( \pm m, \pm m)} \\
y_{( \pm m, \pm m)}
\end{array}\right)=A^{-n}\left(\begin{array}{c} 
\pm m \\
\pm m
\end{array}\right) .
$$

Since the image of $\left\{v:\|v\|_{\infty}=1\right\}=[-1,1]^{2}$ under $A^{-n}$ is a parallelogram,

$$
\left\|A^{-n}\right\|_{\infty}=\max \left\|A^{-n}\left(\begin{array}{c} 
\pm 1 \\
\pm 1
\end{array}\right)\right\|_{\infty}
$$

so

$$
D_{n}^{\prime}\left\|A^{-n}\right\|_{\infty}=\max \left\|A^{-n}\left(\begin{array}{c} 
\pm D_{n}^{\prime} \\
\pm D_{n}^{\prime}
\end{array}\right)\right\|_{\infty}=y_{0} \geq 1
$$

Hence,

$$
\frac{1}{\left\|A^{-n}\right\|_{\infty}} \leq D_{n}^{\prime} \leq \frac{1}{\left\|A^{-n}\right\|_{\infty}}+1
$$

Since $D_{n}=D_{n}^{\prime}$ and $\left\|\bar{A}^{-n}\right\|_{\infty}=\left\|A^{-n}\right\|_{\infty}$, the proof is completed.

Corollary III.17. Let $f$ be a Lattès-type map with orbifold type $(2,2,2,2)$, and let $A$ be its corresponding transformation from $\mathbb{R}^{2}$ to $\mathbb{R}^{2}$. Then the combinatorial expansion factor $\Lambda_{0}(f)$ equals the minimum absolute value of the eigenvalues of $A$. 
Proof. By the previous proposition,

$$
\frac{1}{\left\|A^{-n}\right\|_{\infty}} \leq D_{n} \leq \frac{1}{\left\|A^{-n}\right\|_{\infty}}+1
$$

Taking $n$-th roots gives

$$
\left(\frac{1}{\left\|A^{-n}\right\|_{\infty}}\right)^{1 / n} \leq D_{n}{ }^{1 / n} \leq\left(\frac{1}{\left\|A^{-n}\right\|_{\infty}}+1\right)^{1 / n}
$$

so by Gelfand's formula (see Theorem 13 in [13, Chapter 8]),

$$
\lim _{n \rightarrow \infty} D_{n}^{1 / n}=\lim _{n \rightarrow \infty} \frac{1}{\left\|A^{-n}\right\|_{\infty}^{1 / n}}=\frac{1}{\rho\left(A^{-1}\right)},
$$

where $\rho\left(A^{-1}\right)$ is the spectral radius of $A^{-1}$. On the other hand, the spectral radius of $A^{-1}$ is the maximal absolute value of the eigenvalues of $A^{-1}$, which is equal to $1 /\left|\lambda_{1}\right|$, where $\left|\lambda_{1}\right|$ is the minimum absolute value of the eigenvalues of $A$. We conclude that

$$
\Lambda_{0}=\lim _{n \rightarrow \infty} D_{n}^{1 / n}=\left|\lambda_{1}\right|
$$

Proposition III.18. Let $f$ be a Lattès map over $\Lambda$ and let $\mathcal{C} \subseteq \mathbb{S}^{2}$ be a Jordan curve containing all postcritical points of $f$. Then there exists a constant $c>0$ such that $D_{n}(f, \mathcal{C}) \geq c(\operatorname{deg} f)^{n / 2}$ for all $n>0$.

Proof. First, assume that $f$ is a Lattès map with orbifold type $(2,2,2,2)$. The affine map $A$ is defined over $\mathbb{C}$, i.e., $A: \mathbb{C} \rightarrow \mathbb{C}$. Since $A$ is conformal,

$$
\left\|A v_{1}\right\|_{2}=\left\|A v_{2}\right\|_{2}
$$

if $\left\|v_{1}\right\|_{2}=\left\|v_{2}\right\|_{2}$, where $\|v\|_{2}=\left(x^{2}+y^{2}\right)^{1 / 2}$ for $v=(x, y) \in \mathbb{R}^{2}$. We have that

$$
\|A v\|_{\infty}=\left\|A \frac{v}{\|v\|_{2}}\right\|_{\infty}\|v\|_{2} \geq \operatorname{det}(A)^{1 / 2}\|v\|_{2}
$$


In addition, by the definition of norms we know that

$$
\|v\|_{2} \geq\|v\|_{\infty}
$$

for $v \in \mathbb{R}^{2}$. Hence, we have

$$
\begin{aligned}
\|A\|_{\infty} & =\max \left\{\|A v\|_{\infty}: v \in \mathbb{R}^{2},\|v\|_{\infty}=1\right\} \\
& \geq \max \left\{\operatorname{det}(A)^{1 / 2}\|v\|_{2}\right\} \geq \operatorname{det}(A)^{1 / 2}
\end{aligned}
$$

By Proposition III.16,

$$
D_{n} \geq \frac{1}{\left\|A^{-n}\right\|_{\infty}}=\frac{1}{\operatorname{det}\left(A^{-n}\right)^{1 / 2}}=\operatorname{det}(A)^{n / 2}=\operatorname{deg}(f)^{n / 2}
$$

For Lattès maps with \# post $(f)=3$, by the proof of Lemma III.8, there are only three cases :

$$
(2,3,6),(2,4,4),(3,3,3)
$$

Each of them corresponds to a unique tiling on the plane by triangles $\triangle$ with prescribed angles

$$
\left(\frac{\pi}{2}, \frac{\pi}{3}, \frac{\pi}{6}\right),\left(\frac{\pi}{2}, \frac{\pi}{4}, \frac{\pi}{4}\right),\left(\frac{\pi}{3}, \frac{\pi}{3}, \frac{\pi}{3}\right)
$$

respectively, up to translation and rotation. We refer the reader to Page 13 in [18] for more details.

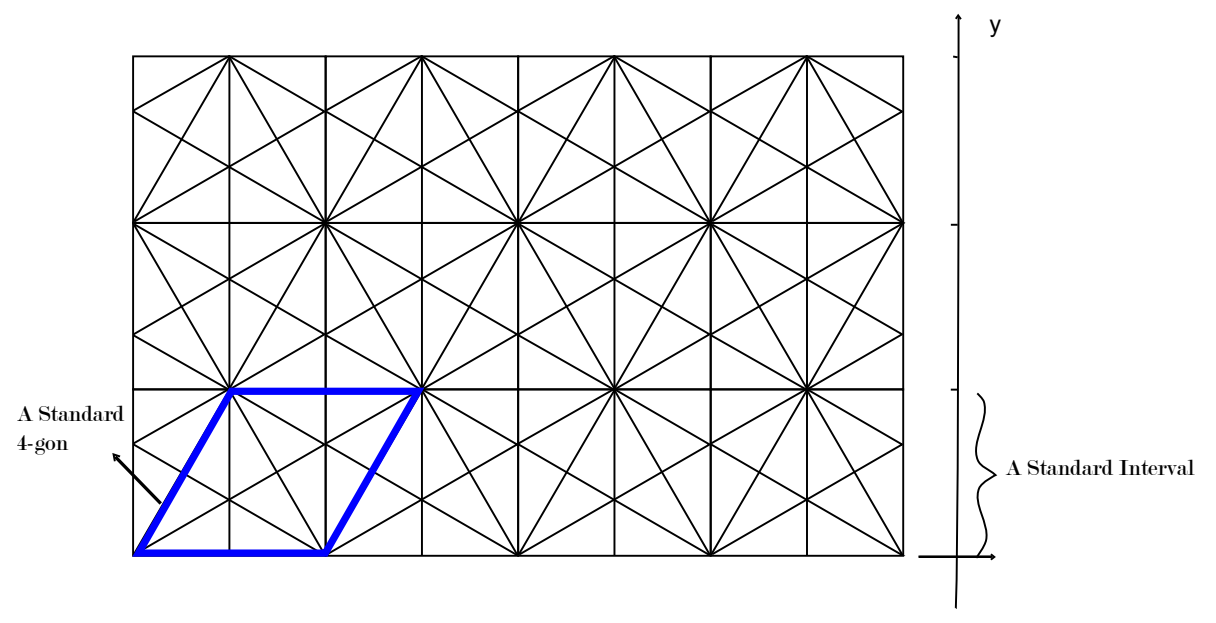


In the case $(2,3,6)$ (see the picture above), we have the relation

$$
D_{n} \leq C_{n} \leq 6 D_{n}
$$

where $C_{n}$ is the minimum number of tiles needed to connect opposite sides of the 4-gon. Since the map $f$ is conformal, any connected component of the preimage of a triangle $\triangle$ under $f^{n}$ is a triangle $\triangle^{\prime}$ similar to $\triangle$ by scalar $s^{-n}=\operatorname{det}(A)^{-n / 2}=$ $\operatorname{deg}(f)^{-n / 2}$, i.e., $\triangle^{\prime}=s^{-n} \triangle$. From the picture, we have $C_{0}=2$, and notice that the minimum number of tiles needed to connect horizontal sides and vertical sides are the same. Scale the standard 4-gon by $s^{n}$. In this process, scaling $\triangle^{\prime}$ by $s^{n}$, we get that $s^{n} \triangle^{\prime}$ is the same size as $\triangle$. If we project the picture onto the $y$-axis, we get $s^{n}$ standard intervals, and each standard interval needs at least two projections of $s^{n} \triangle^{\prime}$ triangles to connect its endpoints. Here a standard interval means an interval on the $y$-axis which is the projection of a standard 4-gon. Hence, $C_{n} \geq 2 s^{n}=2 \operatorname{deg}(f)^{n / 2}$. We get $D_{n} \geq \frac{1}{3} \operatorname{deg}(f)^{n / 2}$.

The other two cases are easier, and can be dealt with similarly.

We conclude that $D_{n} \geq c \operatorname{deg}(f)^{n / 2}$ for some $c>0$ for all Lattès maps.

\subsection{Existence of the Visual Metric}

In this section, we prove that there is a visual metric on $\mathbb{S}^{2}$ with expansion factor equal to $\operatorname{deg}(f)^{1 / 2}$ under the three conditions in Theorem III.41. This will imply that the expanding Thurston map $f$ is topologically conjugate to a Lattès map.

We refer to the following assumptions as $(*)$ :

The map $f: \mathbb{S}^{2} \rightarrow \mathbb{S}^{2}$ is an expanding Thurston map with no

(*) periodic critical points, and let $\mathcal{C}$ be a Jordan curve in $\mathbb{S}^{2}$ that is invariant under $f$ and $\operatorname{post}(f) \subset \mathcal{C}$. 
Notice that the cell decompositions $\mathcal{D}_{n}$ of $\mathbb{S}^{2}$ induced by the Jordan curve above are compatible with each other.

Let $\Lambda_{0}:=(\operatorname{deg} f)^{1 / 2}$. We refer to the following assumptions as $(* *)$ :

The map $f: \mathbb{S}^{2} \rightarrow \mathbb{S}^{2}$ is an expanding Thurston map with no $(* *)$ periodic critical points, and there exists a constant $c>0$ such that $D_{n}=D_{n}(f, \mathcal{C}) \geq c \Lambda_{0}^{n / 2}$ for all $n>0$, where $\mathcal{C}$ is a Jordan curve in $\mathbb{S}^{2}$ that is invariant under $f$ and $\operatorname{post}(f) \subset \mathcal{C}$.

First, let us review some definitions (see the proof of Theorem 17.3 in [3] for more details). Let $f$ be an expanding Thurston map. By Section 2.1, we have a sequence of cell decompositions of the underlying space $\mathbb{S}^{2}$ by tiles. We define a tile chain $P$ to be a finite sequence of tiles

$$
X_{1}, \ldots, X_{N}
$$

such that $X_{j} \cap X_{j+1} \neq \emptyset$ for $j=1, \ldots, N-1$. We also write

$$
P=X_{1} X_{2} \ldots X_{N}
$$

and we use $|P|$ to denote the underlying set $\bigcup_{i=1}^{N} X_{i}$. In addition, if $X_{n}$ intersects with $X_{1}$, then we call the tile chain $P$ a tile loop. For $A, B \subseteq \mathbb{S}^{2}$, we say that the tile chain $P$ joins the sets $A$ and $B$ if

$$
A \cap X_{1} \neq \emptyset \text { and } B \cap X_{N} \neq \emptyset \text {. }
$$

We say that the tile chain $P$ joins the points $x$ and $y$ if $P$ joins $\{x\}$ and $\{y\}$. A subchain of $P=X_{1} X_{2} \ldots X_{N}$ is a tile chain of the form

$$
X_{j_{1}} \ldots X_{j_{s}}, \text { where } 1 \leq j_{1}<\ldots<j_{s} \leq N
$$

We call a tile chain $P=X_{1} X_{2} \ldots X_{N}$ simple if there is no subchain of $P$ that joins $X_{1}$ and $X_{N}$. We call a tile chain $P=X_{1} X_{2} \ldots X_{N}$ an $n$-tile chain if all the tiles $X_{i}$ 
are $n$-tiles $1 \leq i \leq N$. An $n$-tile chain $P=X_{1} X_{2} \ldots X_{N}$ is called an $e$-chain if there exists an $n$-edge $e_{i}$ with $e_{i} \subseteq X_{i} \cap X_{i+1}$ for $i=1, \ldots, N$. The $e$-chain $j$ oins the tiles $X$ and $Y$ if $X_{1}=X$ and $X_{N}=Y$. A set $M$ of $n$-tiles is e-connected if every two tiles in $M$ can be joined by an $e$-chain.

The following lemma is from [3, Lemma 14.4].

Lemma III.19. Let $\gamma \subset \mathbb{S}^{2}$ be a path in $\mathbb{S}^{2}$ defined on a closed interval $J \subset \mathbb{R}$ and $M=M(\gamma)$ be the set of tiles having nonempty intersection with $\gamma$. Then $M$ is e-connected.

If $P=X_{1} \ldots X_{N}$ is a tile chain, then we define the length of the tile chain to be the number of tiles in $P$ :

$$
\operatorname{length}(P)=N
$$

For $n \geq 1$, we define a function

$$
d_{n}: \mathbb{S}^{2} \times \mathbb{S}^{2} \rightarrow \mathbb{R}
$$

as follows: for any $x, y \in \mathbb{S}^{2}$, if $x=y$, then $d_{n}(x, y)=0$; otherwise,

$$
d_{n}(x, y)=\min \{\operatorname{length}(P)\} \Lambda^{-n},
$$

where the minimum is taken over all $n$-tile chains $P$ joining $x$ and $y$. It is not hard to check that $d_{n}$ is a metric.

In the following, we will show that for any $x, y \in \mathbb{S}^{2}$ with $x \neq y$, the ratio

$$
d_{n}(x, y) / \Lambda^{-m(x, y)}
$$

has a uniform upper and lower bound for all $n>m(x, y)$ (see Lemma III.29 and Lemma III.30). Then we will define a distance function

$$
d=\limsup _{n \rightarrow \infty} d_{n}
$$


and we will see that this metric $d$ is a visual metric on $\mathbb{S}^{2}$ with expansion factor $\Lambda$ (see Proposition III.31).

Definition III.20. For $n \geq 3$, we call a topological space $X$ an $n$-gon if $X$ is homeomorphic to the closed unit disk $\mathbb{D} \subset \mathbb{R}^{2}$ with $n$ points marked on the boundary of $X$. Since the boundary of an $n$-gon is homeomorphic to $\mathbb{S}^{1}$, there is a natural cyclic order for the $n$ marked points on the boundary. We call these $n$-points vertices of the $n$-gon and the parts of the boundary of $X$ joining two consecutive vertices in the cyclic order edges of the $n$-gon.

Now let us review some basic definitions from graph theory (we refer the reader to [6] for more details). A graph $G$ is a pair $(V, E)$ of sets such that the edge set $E=E(G)$ is a symmetric subset of the Cartesian product $V \times V$ of the vertex set $V=V(G)$. We call a graph $G^{\prime}=\left(E^{\prime}, V^{\prime}\right)$ a subgraph of $G=(V, E)$ if

$$
E^{\prime} \subseteq E \text { and } V^{\prime} \subseteq V
$$

written as $G^{\prime} \subseteq G$. A (simple) path in a graph $G=(V, E)$ is a non-empty subgraph $P=\left(V^{\prime}, E^{\prime}\right)$ of the form

$$
V^{\prime}=V^{\prime}(P)=\left\{x_{0}, x_{1}, \ldots, x_{k}\right\}
$$

and

$$
E^{\prime}=E^{\prime}(P)=\left\{x_{0} x_{1}, x_{1} x_{2}, \ldots, x_{k-1} x_{k}\right\},
$$

where the $x_{i} \in V^{\prime}$ are all distinct, and for $u, v \in V$, uv denotes the edge with end points $u$ and $v$.

$x_{i} x_{i+1}$ denotes an edge in $G$ for $i=0, \ldots, k-1$. We also write a path as

$$
P=x_{0} x_{1} \ldots x_{k}
$$


and call $P$ a path from $x_{0}$ to $x_{k}$. Given sets $A, B$ of vertices in $G$, we call $P=$ $x_{0} x_{1} \ldots x_{k}$ an $A-B$ path if

$$
x_{0} \in V(P) \cap A \quad \text { and } \quad x_{k} \in V(P) \cap B .
$$

Given a graph $G=(V, E)$, if $A, B, X \subseteq V$ are such that every $A$-B path in $G$ contains a vertex from $X$, we say that $X$ separates the sets $A$ and $B$ in $G$. We call $X$ a separating set for $A$ and $B$. We will use the following theorem (see Theorem 3.3.1 in [6]).

Theorem III.21 (Menger's theorem). Let $G=(V, E)$ be a finite graph and $A, B \subseteq$ $V$. Then the minimal cardinality of a set separating $A$ and $B$ in $G$ is equal to the maximal number of disjoint $A-B$ paths in $G$.

Proposition III.22. Let $f$ be a Thurston map and $\mathcal{C} \subseteq \mathbb{S}^{2}$ be a Jordan curve that is invariant under $f$ and contains post $(f)$. There exists a constant $C>0$, such that

$$
D_{n}=D_{n}(f, \mathcal{C}) \leq C \operatorname{deg}(f)^{n / 2}
$$

for all $n \geq 0$.

Proof. First, assume that $m=\#$ post $(f) \geq 4$. Let $e_{1}, \ldots, e_{m}$ be 0 -edges in cyclic order. Let $P_{n}$ be an $n$-tile chain consisting of $D_{n} n$-tiles joining opposite sides of the Jordan curve $\mathcal{C}$. Without loss of generality, we may assume that $P_{n}$ joins nonadjacent edges $e_{1}$ and $e_{l}$, for some $2<l<m$. There are two cases: either $\left|P_{n}\right|$ is a subset of one of the 0-tiles, or $\left|P_{n}\right|$ intersects with the interior of both 0-tiles, and $e_{1}$ and $e_{l}$ share an adjacent edge, which is the only edge intersecting with $\left|P_{n}\right|$ besides $e_{1}$ and $e_{l}$. Indeed, suppose that $\left|P_{n}\right|$ is not a subset of one of the 0-tiles. If $\left|P_{n}\right|$ intersects with more than one adjacent edges of $e_{1}$ and $e_{l}$ or with a non-adjacent 
edge of $e_{1}$ or $e_{l}$, then we have a strictly shorter $n$-tile chain joining opposite sides of the Jordan curve $\mathcal{C}$, which is a contradiction to the definition of $D_{n}$.

For the first case, the tile chain $P_{n}$ splits one of the 0-tile (an $m$-gon) into two parts, and pick an 0-edge on either part, and call them $a$ and $b$ respectively. For the second case, without loss of generality, we assume that $P_{n}$ is an $n$-tile chain consisting of $D_{n}$ tiles joining opposite edges $e_{1}$ and $e_{3}$ (possibly intersecting with $e_{2}$ ). We cut along all the edges except edge $e_{2}$, where one edge becomes two, and unfold the cut sphere to get a $(2 m-2)$-gon. Another way to think of this $(2 m-2)$-gon is by gluing two copies of an $m$-gon along the edge $e_{2}$. Notice that $P_{n}$ divides this $(2 m-2)$-gon into two parts. Pick an edge on either part which is not $e_{1}$ or $e_{3}$, and call them $a$ and $b$ respectively. Let $A$ be the set of all $n$-tiles intersecting with $a$, and $B$ be the set of all $n$-tiles intersecting with $b$. Since the set $A$ and $B$ lie on different sides of $P_{n}, P_{n}$ separates $A$ and $B$. Consider the dual graph associated with the $n$-tile decomposition. By Menger's theorem, there are at least $D_{n}$ many disjoint $A-B$ paths. Let $N_{n}$ be the minimum number of tiles in an $A-B$ path, and since an $A$ - $B$ path is an $n$-tile chain joining opposite sides of the Jordan curve $\mathcal{C}$, we have $D_{n} \leq N_{n}$. We get

$$
D_{n}^{2} \leq D_{n} N_{n} \leq 2(\operatorname{deg}(f))^{n}
$$

SO

$$
D_{n} \leq C \operatorname{deg}(f)^{n / 2}
$$

for $C=\sqrt{2}$.

When \#post $(f)=3$, we can cut along any two edges of the 3-gons, and we unfold it to get a 4 -gon. Let $P_{n}$ be an $n$-tile chain joining non-adjacent edges of this 4 -gon, with the minimum number of $n$-tiles. Let $C_{n}=\left|P_{n}\right|$. Since $P_{n}$ also joins opposite sides of the Jordan curve $\mathcal{C}$, we have $D_{n} \leq\left|P_{n}\right|=C_{n}$. By the same argument for 
\#post $(f) \geq 4$, we have $C_{n} \leq C \operatorname{deg}(f)^{n / 2}$ for some $C>0$. Hence, $D_{n} \leq C \operatorname{deg}(f)^{n / 2}$ for some $C>0$.

Let $X$ be a union of $m$-gons, and let $\mathcal{D}$ be a cell decomposition of $X$ by $m$-gons. We define a graph $G(\mathcal{D})$ with vertex set $V$ being the set of $m$-gons, and with an edge between two vertices if and only if the corresponding $m$-gons share a common edge. We call $G(\mathcal{D})$ the dual graph associated with cell decomposition $\mathcal{D}$.

Given an $l$-vertex $v$, let $W \subset \mathbb{S}^{2}$ be the union of $l$-tiles containing $v$, then by Lemma II.6,

$$
W=\overline{W^{l}(v)}
$$

where $W^{l}(v)$ is the $l$-flower of $v$. Recall $W(v)$ is the union of the interior of $n$-cells intersecting with $v$, and $W^{l}(v)$ is connected. For $n>l$, let $\mathcal{D}^{n}(v)$ be the set of $n$-cells contained in $W$. This gives us a cell-decomposition of $W$. Denote $G^{n}(v)$ be the dual graph associated with the cell decomposition $\mathcal{D}^{n}(v)$. Let $M^{n}(v)$ be the set of $n$-tiles in $W$ that are non-disjoint from the boundary $\partial W$ of $W$.

Lemma III.23. Assume that $(*)$ satisfies and $n>l \geq 0$. Use the notation as above. The graph $G^{n}(v)$ is path connected and the set $M^{n}(v)$ is e-connected.

Proof. By Lemma II.6, the $l$-flower $W^{l}(v)$ is simply connected. There exists a path $\gamma \subset W^{l}(v)$ containing all $n$-vertices in $W^{l}(v)$. Let $V=V(\gamma)$ be the set of tiles having nonempty intersection with $\gamma$. Then $V$ is the vertex set of the graph $G^{n}(v)$. Lemma III.19 states that $V$ is $e$-connected, which implies that $G^{n}(v)$ is connected.

Any two tiles intersecting with $\partial W$ can be joined by a finite sequence of $n$-edges in $\partial W$. In order to show that $M^{n}(v)$ is $e$-connected, we only need to show that for any $X, Y \in M^{n}(v)$, with an $n$-edge $e \subset \partial W$ such that

$$
X \cap e \neq \emptyset \text { and } Y \cap e \neq \emptyset \text {, }
$$


$X$ and $Y$ can be joined by an $e$-chain in $M^{n}(v)$. The $n$-tile $Z \in M^{n}(v)$ containing $\bar{e}$ shares common vertices with $X$ and $Y$ respectively. So there are $e$-chains joining $X$ and $Z, Z$ and $Y$ respectively, and there is an $e$-chain joining $X$ and $Y$. Hence, the set $M^{n}(v)$ is e-connected.

Given a set $T$, let $A, B \subset U$ such that

$$
A \cap B=\emptyset
$$

We say that a set $U \subset T$ separates $A$ and $B$ if for any path $\gamma \subseteq T$ joining $A$ and $B$,

$$
\gamma \cap U \neq \emptyset
$$

We call $U$ a separating set of $A$ and $B$ in $T$. For $x, y \in T$, we call $U$ a separating set of $x$ and $y$ in $T$ if $U$ separates $\{x\}$ and $\{y\}$ in $T$.

Lemma III.24. Assume that $(*)$ satisfies and $n>l+1>0$. Let $v$ be an l-vertex. Let $X$ and $Y$ be $(l+1)$-tiles in $\overline{W^{l}(v)}$, and let $A$ and $B$ the set of $n$-tiles in $X$ and $Y$ respectively. Let $S$ be a separating set of $A$ and $B$ in $G^{n}(v)$. Then the union $U$ of all $n$-tiles in $S$ separates $X$ and $Y$ in $W^{l}(v)$.

For the meaning of notations involved in the lemma above, please see the paragraph before Lemma III.23.

Proof. Assume that the set $U$ does not separate $X$ and $Y$ in $W^{l}(v)$. Then there exists a path $\gamma \subset W^{l}(v)$ joining $X$ and $Y$ such that

$$
\gamma \cap U=\emptyset
$$

By Lemma 14.4 in [3], the set $M(\gamma)$ of $n$-tiles intersecting with $\gamma$ is $e$-connected. In addition, we have that

$$
M(\gamma) \cap S=\emptyset
$$


since $\gamma \cap U=\emptyset$. This means that there exists an $e$-path in $M(\gamma) \backslash S \subset G^{l}(v)$ joining $A$ and $B$. This is a contradiction of definition of separating set $S$. Hence, the union $U$ of all $n$-tiles in $S$ separates $X$ and $Y$ in $W^{l}(v)$.

The following theorem is from [19, Page 110].

Theorem III.25 (Janiszewski). Let $A$ and $B$ be closed subset of $\mathbb{S}^{2}$, and $A \cap B$ is connected. For $x, y \in \mathbb{S}^{2}$, if neither $A$ nor $B$ separates $x$ and $y$, then $A \cup B$ does not separate $x$ and $y$.

As a corollary of Janiszewski Theorem, we have the following.

Corollary III.26. Let $U$ be a closed subset of $\mathbb{S}^{2}$ with finitely many connected components. For $x, y \in \mathbb{S}^{2}$, if the set $U$ separates $x$ and $y$, then one of the connected component of $U$ separates $x$ and $y$.

Proof. This follows from Janiszewski Theorem by induction on the number of connected components of $U$.

Lemma III.27. Let $W$ be a simply connected region in $\mathbb{S}^{2}$. Let $U$ be a closed subset of the closure $\bar{W}$ in $\mathbb{S}^{2}$ with finitely many connected components. For $x, y \in W$, if $U$ separates $x$ and $y$ in $W$, then there exists a connected component of $U$ separating $x$ and $y$.

Proof. Without loss of generality, we may assume that $U=A \cup B$ where $A$ and $B$ are connected components of $U$. Let $\partial W$ be the boundary of $W$ in $\mathbb{S}^{2}$. For any path $\gamma \subseteq \mathbb{S}^{2}$ from $x$ to $y$, if $\gamma \subset W$

$$
\gamma \cap U \neq \emptyset
$$

if $\gamma \not \subset W$,

$$
\gamma \cap \partial W \neq \emptyset .
$$


So the set $U \cup \partial W$ separates $x$ and $y$ in $\mathbb{S}^{2}$.

Case 1 neither $A$ nor $B$ intersects with $\partial W$. Since $\partial W$ does not separates $x$ and $y$ in $\mathbb{S}^{2}$, Corollary ?? implies that either the set $A$ or $B$ separates $x$ and $y$ in $\mathbb{S}^{2}$.

Case 2 both $A$ and $B$ intersect with $\partial W$. Let

$$
A^{\prime}=A \cup \partial W \text { and } B^{\prime}=B \cup \partial W \text {. }
$$

Notice that $A^{\prime} \cap B^{\prime}=\partial W$ is connected. We claim that either the set $A^{\prime}$ or $B^{\prime}$ separates $x$ and $y$ in $\mathbb{S}^{2}$. If neither $A^{\prime}$ nor $B^{\prime}$ separates $x$ and $y$ in $\mathbb{S}^{2}$, then by Theorem Jankowski, the set

$$
A^{\prime} \cup B^{\prime}=U \cup \partial W
$$

does not separates $x$ and $y$, which is a contradiction. Without loss of generality, assume that $A^{\prime}$ separates $x$ and $y$ in $\mathbb{S}^{2}$. Then $A$ separates $x$ and $y$ in $W$.

Case 3 only $A$ or $B$ intersects with $\partial W$. Without loss of generality, assume that

$$
A \cap \partial W \neq \emptyset \text { and } B \cap \partial W=\emptyset .
$$

Let $A^{\prime}=A \cup \partial W$. By Corollary ??, either $A^{\prime}$ or $B$ separates $x$ and $y$ in $\mathbb{S}^{2}$. This implies that either $A$ or $B$ separates $x$ and $y$ in $U$.

Hence, one of the connected component of $U$ separates $x$ and $y$ in $U$.

Given an $h$-tile $X^{h}$, and an $(h-1)$-tile $X^{h-1}$, if $X^{h} \subset X^{h-1}$, then we call $X^{h-1}$ the parent of $X^{h}$.

Lemma III.28. Assume that (**) satisfies and $n>h>0$. Let $X^{h}, Y^{h}$ be disjoint $h$-tiles and let $X^{h-1}, Y^{h-1}$ be their parents respectively. Assume $X^{h-1} \cap Y^{h-1} \neq \emptyset$. There exists an $n$-tile chain with at most $c^{\prime} \Lambda_{0}^{n-h}$ many tiles joining $X^{h}$ and $Y^{h}$, where $c^{\prime}>0$ only depends on $f$. 
Proof. Let $v$ be an $(h-1)$-vertex in $X^{h-1} \cap Y^{h-1}$, and let $G^{n}(v)$ be the dual graph associated with $W^{h-1}(v)$ (see the paragraph before Lemma III.23 for the meaning of the notations). By Lemma III.23, the graph $G^{n}(v)$ is path-connected.

Let $A$ be all the $n$-tiles in $X^{h}$, and $B$ be all the $n$-tiles in $Y^{h}$. Let $S$ be a minimal separating set between $A$ and $B$. Let $U$ be the union of the underlying tiles in S. By Lemma III.24, the set $U$ separates $X^{h}$ and $Y^{h}$. Since $X^{h}$ and $Y^{h}$ are both contractible, by Lemma III.27, one of the connected component of $U$ separates $X^{h}$ and $Y^{h}$. Since $S$ is a minimal separating set, the separating set $U$ is connected.

We claim that the set $U$ joins non-adjacent $h$-edges. Assume that the set $U$ only intersects with adjacent $h$-edges. If there is no $h$-edge or only one $h$-edge intersecting with $U$, then we can pick an $h$-vertex, and call it $w$, such that

$$
U \subset W^{h}(w)
$$

Otherwise, the $h$-edges non-disjoint from $U$ share a common $h$-vertex, and call this vertex $w$. So

$$
U \subset W^{h}(w)
$$

By Lemma III.23, the set $M^{n}(w)$ of all $n$-tiles non-disjoint from $\partial W^{h}(w)$ is $e$ connected. Since $S$ is a set of $n$-tiles disjoint from $\partial W^{h}(w)$, we have that

$$
M^{n}(w) \cap S=\emptyset .
$$

Fix an $n$-tile $X^{n} \subset X^{h}$ such that $X^{n} \in M^{n}(w)$ if $X^{h} \cap W^{h}(w) \neq \emptyset$. Fix an $n$ tile $Y^{n} \subset Y^{h}$ such that $Y^{n} \in M^{n}(w)$ if $Y^{h} \cap W^{h}(w) \neq \emptyset$. There exists a path $P=X_{1} \ldots X_{l}$ joining $X^{n}$ and $Y^{n}$ in $G^{n}(v)$. If

$$
P \cap S \neq \emptyset
$$


let $X_{i}, X_{j} \in P$ be the first and last $n$-tile in $M^{n}(w)$, so there is a path $P^{\prime} \subset M^{n}(v)$ joining $X_{i}$ and $X_{j}$. Then the new path

$$
\bar{P}=X_{1} \ldots X_{i} P^{\prime} X_{j} \ldots X_{l}
$$

disjoint from $S$ joins $X^{n}$ and $Y^{n}$ in $G^{n}(v)$. This is a contradiction to the definition of separating set $S$. Hence, our claim is true and there exist at least $D_{n-h}$ many $n$-tiles in $S$.

Let $N_{n}$ be the minimal number of $n$-tiles in an $A$ - $B$ path. By Menger's Theorem, there are at lease $D_{n-h}$ many non-disjoint $A-B$ paths in $G_{n}$. Thus

$$
N_{n} D_{n-h} \leq N(\operatorname{deg} f)^{(n-h)},
$$

where $N>0$ is a constant as in Lemma II.8 which only depends on $f$. Hence

$$
N_{n} \leq \frac{N(\operatorname{deg} f)^{(n-h)}}{D_{n-h}} \leq \frac{N}{C} \Lambda_{0}^{n-h}=c^{\prime} \Lambda_{0}^{n-h},
$$

where $c^{\prime}$ only depends on $f$.

Lemma III.29. Assume that $(* *)$ satisfies and assume that $\Lambda_{0}>2$. There exists a constant $C>0$ only depending on $f$, such that for any $x, y \in \mathbb{S}^{2}$ with $x \neq y$ and for any $n>m(x, y)$,

$$
d_{n}(x, y) \leq C \Lambda_{0}^{-m(x, y)}
$$

Proof. For simplicity of notation, denote $l=\frac{1}{2}$, and denote an $n$-tile chain $P=$ $X_{1} \ldots X_{N}$ as $P\left(X_{1}, X_{N}\right)$. Let $m=m(x, y)$, and let $X_{0}, Y_{1}$ be $n$-tiles containing $x$ and $y$ respectively.

The idea of the proof is as follows: We will build an $n$-tile chain joining $x$ and $y$ that is "not too long". First construct an $n$-tile chain $P_{l}$ joining two $m$-tiles containing $x$ and $y$ respectively. There are 2 gaps between $x$ and the first tile in $P_{l}$, and between 
the last tile in $P_{l}$ and $y$. We try to fill in the gaps by constructing two $n$-tile chains $P_{l^{2}}, P_{3 l^{2}}$ joining the $(m+1)$-tiles containing $x$ (the head of the first gap) and the first tile in $P_{l}$ (the tail of the first gap), and joining the $(m+1)$-tiles containing the last tile in $P_{l}$ (the head of the second gap) and $y$ (the tail of the second gap). We now have 4 new smaller gaps. We try to fill them again by the similar method as above. Namely, construct $n$-tile chains joining $(m+2)$-tiles containing the head and the tail of each gap. Keep on trying to fill the gaps between $(m+t)$-tiles with $t$ getting larger each step. Eventually, we will have $m+t=n$, and there are $2^{n-m}$ many gaps. We can then fill all the gaps between $n$-tiles by $n$-chains, and hence obtain our desired $n$-chain.

Let $X^{m}$ and $Y^{m}$ be the disjoint $m$-tiles containing $X_{0}$ and $Y_{1}$ respectively. By the definition of $m$, there exists two non-disjoint $(m-1)$-tiles that are parents of $X^{m}$ and $Y^{m}$ respectively. By Lemma III.28, there exists an $n$-tile chain $P_{l}=P\left(Y_{l}, X_{l}\right)$ with at most $c^{\prime} \Lambda_{0}^{n-m}$ tiles joining $X^{m}$ and $Y^{m}$, where $c^{\prime}>0$ is a constant only depending on $f$.

Let $X_{0}^{m+1}, Y_{l}^{m+1}, X_{l}^{m+1}, Y_{1}^{m+1}$ be the $(m+1)$-tiles containing the $n$-tiles $X_{0}, Y_{l}, X_{l}, Y_{1}$ respectively. The parents of $X_{0}^{m+1}, Y_{l}^{m+1}$ are non-disjoint. By Lemma III.28, there exists an $n$-tile chain $P_{l^{2}}=P\left(Y_{l^{2}}, X_{l^{2}}\right)$ with at most $c^{\prime} \Lambda_{0}^{n-m-1}$ many tiles joining $X_{0}^{m+1}$ and $X_{l}^{m+1}$. Similarly, there exists an $n$-tile chain $P_{3 l^{2}}=P\left(Y_{3 l^{2}}, X_{3 l^{2}}\right)$ with at most $c^{\prime} \Lambda_{0}^{n-m-1}$ tiles joining $X_{l}^{m+1}$ and $Y^{m+1}$.

Continuing in this manner, for $0 \leq t \leq n-m$, we let $X_{i l^{t}}^{m+t}, Y_{(i+1) l^{t}}^{m+t}$ be the $(m+t)$ tiles containing the $n$-tiles $X_{i l^{t}}, Y_{(i+1) l^{t}}$ respectively, for $i=0,1, \ldots, 2^{t}-1$. The parents of $X_{i l^{t}}^{m+t}, Y_{(i+1) l^{t}}^{m+t}$ are non-disjoint, so by Lemma III.28, there exists an $n$-tile chain

$$
P_{(2 i+1) l^{t+1}}=P\left(Y_{(2 i+1) l^{t+1}}, X_{(2 i+1) l^{t+1}}\right)
$$


with at most $c^{\prime} \Lambda_{0}^{n-m-t}$ many tiles joining $X_{i l^{t}}^{m+t}$ and $Y_{(i+1) l^{t}}^{m+t}$, for $i=0,1, \ldots, 2^{t}-1$.

In particular, when $t=n-m$, the $n$-tiles $X_{i l^{n-m}}^{n}, Y_{(i+1) l^{n-m}}^{n}$ contain the $n$-tiles $X_{i l^{n-m}}, Y_{(i+1) l^{n-m}}$ respectively, for $i=0,1, \ldots, 2^{n-m}-1$, and there exists an $n$-tile chain

$$
P_{(2 i+1) l^{n-m+1}}=P\left(Y_{(2 i+1) l^{n-m+1}}, X_{(2 i+1) l^{n-m+1}}\right)
$$

with at most $c^{\prime}$ tiles joining $X_{i l^{n-m}}^{n}$ and $Y_{(i+1) l^{n-m}}^{n}$, for $i=0,1, \ldots, 2^{n-m}-1$. Notice that

$$
X_{i l^{n-m}}^{n}=X_{i l^{n-m}}=Y_{(2 i+1) l^{n-m+1}}
$$

and

$$
Y_{(i+1) l^{n-m}}^{n}=Y_{(i+1) l^{n-m}}=X_{(2 i+1) l^{n-m+1}}
$$

for $i=0,1, \ldots, 2^{n-m}-1$, since they are all $n$-tiles. Hence, we get a finite sequence of $n$-tile chains

$$
P_{i l^{n-m+1}}=P\left(Y_{i l^{n-m+1}}, X_{i l^{n-m+1}}\right) \text { for } i=0,1 \ldots, 2^{n-m+1}-1 \text {, }
$$

such that their union joins $X_{0}$ and $Y_{1}$.

This implies that we get an $n$-tile chain $P$ joining $x$ and $y$ with the number of tiles equal to

$$
\begin{aligned}
\operatorname{length}(P)= & \sum_{i=0}^{2^{n-m+1}-1} \operatorname{length}\left(P_{i l^{n-m+1}}\right) \\
= & \operatorname{length}\left(P_{l}\right)+\left(\operatorname{length}\left(P_{l^{2}}\right)+\operatorname{length}\left(P_{3 l^{2}}\right)+\ldots\right. \\
& \quad+\sum_{i=0}^{i=2^{t}-1} \operatorname{length}\left(P_{(2 i+1) l^{t+1}}\right)+\ldots+\sum_{i=0}^{2^{n-m}-1} \operatorname{length}\left(P_{(2 i+1) l^{n-m+1}}\right) \\
\leq & c^{\prime} \Lambda_{0}^{n-m}+2 c^{\prime} \Lambda_{0}^{n-m-1}+\ldots+2^{t} c^{\prime} \Lambda_{0}^{n-m-t}+\ldots+2^{n-m} c^{\prime} \\
= & c^{\prime} \Lambda_{0}^{n-m}\left[1+2 / \Lambda_{0}+\ldots+\left(2 / \Lambda_{0}\right)^{t}+\ldots+\left(2 / \Lambda_{0}\right)^{n-m}\right] \\
\leq & C \Lambda_{0}^{n-m}
\end{aligned}
$$


where $C>0$ only depends on $f$. Therefore, we have

$$
d_{n}(x, y) \leq C \Lambda_{0}^{n-m} \Lambda_{0}^{-n}=C \Lambda_{0}^{-m}=C \Lambda_{0}^{-m(x, y)} .
$$

Lemma III.30. Assume that $(*)$ satisfies. For any $x, y \in \mathbb{S}^{2}$ with $x \neq y$, for any $n>m(x, y)$, we have

$$
d_{n}(x, y) \geq \rho(x, y)
$$

Proof. Let $m=m(x, y)$, and let $X^{m}$ and $Y^{m}$ be disjoint $m$-tiles containing $x$ and $y$ respectively. The length of any $n$-tile chain joining $X^{m}$ and $Y^{m}$ is at least $D_{n-m}$. Hence, we have that

$$
d_{n}(x, y) \geq D_{n-m} \Lambda_{0}^{-n} \geq \Lambda_{0}^{n-m} \Lambda_{0}^{-n}=\Lambda_{0}^{-m}=\Lambda_{0}^{-m(x, y)} .
$$

Proposition III.31. Let $f: \mathbb{S}^{2} \rightarrow \mathbb{S}^{2}$ be an expanding Thurston map with no periodic critical points. Assume there exists $c>0$ such that $D_{n}=D_{n}(f, \mathcal{C}) \geq c(\operatorname{deg} f)^{n / 2}$ for all $n>0$, where $\mathcal{C}$ is a Jordan curve containing post $(f)$. Then there exists a visual metric with

$$
\Lambda=\Lambda_{0}:=(\operatorname{deg} f)^{1 / 2}
$$

as the expansion factor.

See Definition II.11, for the definition of a visual metric.

Proof. By Theorem II.2, for some $n>0$, there exists a Jordan curve $\mathcal{C}$ containing post $(f)$ that is invariant under $f^{n}$. Proposition $8.8(\mathrm{v})$ in [3] states that a metric is a visual metric for $f^{n}$ if and only if it is a visual metric for $f$. Hence, we may assume 
that there exists a Jordan curve $\mathcal{C}$ that is invariant under $f$. Since we take an iterate of $f$, we may assume that

$$
\Lambda_{0}=\Lambda(f)=(\operatorname{deg} f)^{1 / 2}>2 .
$$

Let

$$
d=\limsup _{n \rightarrow \infty} d_{n}
$$

where $d_{n}$ is defined in equation (4.2). We will show that $d$ is a visual metric on $\mathbb{S}^{2}$ with expansion factor $\Lambda_{0}$. The function $d$ is a metric since $d_{n}$ is metric on $\mathbb{S}^{2}$ for all $n>0$.

Fix $x, y \in S^{2}$ such that $x \neq y$. By Lemma III.29,

$$
d(x, y)=\limsup _{n \rightarrow \infty} d_{n}(x, y) \leq C \Lambda_{0}^{-m(x, y)}
$$

where $C>0$ only depends on $f$. By Lemma III.30,

$$
d(x, y)=\limsup _{n \rightarrow \infty} d_{n}(x, y) \geq \Lambda_{0}^{-m(x, y)} .
$$

Therefore, the function $d$ is a visual metric on $\mathbb{S}^{2}$ with expansion factor $\Lambda_{0}=$ $(\operatorname{deg} f)^{1 / 2}$.

\subsection{The Sufficiency of the Conditions}

In this section, we show that under the conditions in Theorem III.41, the expanding Thurston map $f$ is topologically conjugate to a Lattès map.

For the next definition, we use the notion of continuum, which is a compact connected set consisting of more than one point.

Definition III.32. A metric space $(X, d)$ is called linearly locally connected (denote LLC) if there exists some $\lambda>1$ such that the following two conditions are satisfied: 
(LLC1) If $B(a, r)$ is a ball in $X$ and $x, y \in B(a, r)$, then there exists a continuum $E \subseteq B(a, \lambda r)$ containing $x$ and $y$

(LLC2) If $B(a, r)$ is a ball in $X$ and $x, y \in X \backslash B(a, r)$, then there exists a continuum $E \subseteq X \backslash B(a, r / \lambda)$ containing $x$ and $y$

A metric space $X$ is called Ahlfors $Q$-regular for $Q>0$, if for any $x \in X$, $0<r \leq \operatorname{diam} X$, there exists a (Borel) measure $\mu$ such that

$$
\frac{1}{C} r^{Q} \leq \mu(\bar{B}(x, r)) \leq C r^{Q}
$$

where $C \geq 1$ is independent $x$ and $r$. Two metric space $\left(X, d_{X}\right)$ and $\left(Y, d_{Y}\right)$ are quasisymmetriclly equivalent if there are homeomorphisms $f: X \rightarrow Y$ and $\eta:[0, \infty) \rightarrow$ $[0, \infty)$ such that for all $x, y, z \in X$ with $x \neq z$, we have

$$
\frac{d_{Y}(f(x), f(y))}{d_{Y}(f(x), f(z))} \leq \eta\left(\frac{d_{X}(x, y)}{d_{X}(x, z)}\right)
$$

We have a natural metric on $\widehat{\mathbb{C}}=\mathbb{C} \cup\{\infty\}$ by stereographic projection, called the chordal metric, defined by

$$
\delta(z, w)=\frac{2|z-w|}{\sqrt{1+|z|^{2}} \sqrt{1+|w|^{2}}}
$$

and

$$
\delta(z, \infty)=\delta(\infty, z)=\frac{2}{\sqrt{1+|z|^{2}}}
$$

for $z, w \in \mathbb{C}$

Proposition III.33. If we let $d$ be a visual metric that we get under the assumption of Proposition III.31, then $\left(\mathbb{S}^{2}, d\right)$ is Ahlfors 2-regular and quasisymmetrically equivalent to the Riemann sphere $\widehat{\mathbb{C}}$.

Proof. Proposition 19.10 in [3] states that for an expanding Thurston map $f: \mathbb{S}^{2} \rightarrow \mathbb{S}^{2}$ without periodic critical points, if $d$ is a visual metric with expansion factor $\Lambda$, then 
$\left(\mathbb{S}^{2}, d\right)$ is Ahlfors $Q$-regular with

$$
Q=\frac{\log (\operatorname{deg}(f))}{\log \Lambda}
$$

Since our $\Lambda=\operatorname{deg}(f)^{1 / 2}$, the metric space $\left(\mathbb{S}^{2}, d\right)$ is Ahlfors 2-regular.

Proposition 16.3 (iii) in [3] states that $\mathbb{S}^{2}$ with a visual metric $d$ of $f$, is linearly locally connected.

Now our proposition follows immediately from Theorem 1.1 in [2], which states that for a metric space $X$ homeomorphic to $\mathbb{S}^{2}$, if $X$ is linearly locally connected and Ahlfors 2-regular, then $X$ is quasisymmetrically equivalent to the Riemann sphere $\widehat{\mathbb{C}}$.

Theorem 1.7 in [3] states that:

Theorem III.34 (Bonk-Meyer 2010). For an expanding Thurston map with visual metric $d,\left(\mathbb{S}^{2}, d\right)$ is quasisymmetrically equivalent to the Riemann sphere $\widehat{\mathbb{C}}$ if and only if $f$ is topologically conjugate to a rational map.

By this theorem and Proposition III.33, there exists a rational map $R: \mathbb{S}^{2} \rightarrow \mathbb{S}^{2}$ and a homeomorphism $\phi$ such that $\phi \circ f=R \circ \phi$. See the diagram below:

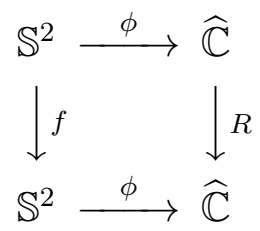

Since $\phi$ is a homeomorphism, $\operatorname{deg}(R)=\operatorname{deg}(f)$. The Jordan curve $\mathcal{C}^{\prime}=\phi(\mathcal{C})$ contains all the post-critical points of $R$, where $\mathcal{C} \subset \mathbb{S}^{2}$ is a Jordan curve containing post $(f)$. Also, $X$ is an $n$-tile under the cell decomposition of $(f, \mathcal{C})$ if and only if $\phi(X)$ is an $n$-tile under the cell decomposition of $\left(R, \mathcal{C}^{\prime}\right)$. So the minimal numbers of $n$-tiles needed to join opposite sides of the Jordan curves are the same, i.e., $D_{n}(R)=D_{n}(f)$. By Proposition III.31 and Proposition III.33, there exists a visual metric $d_{R}$ on $\widehat{\mathbb{C}}$ 
with expansion factor $\Lambda_{0}=(\operatorname{deg}(R))^{1 / 2}$ and $\left(\widehat{\mathbb{C}}, d_{R}\right)$ is Ahlfors 2-regular. In addition, with the metric

$$
d_{R}(\phi(x), \phi(y))=d(x, y),
$$

$\phi$ is an isometry since $m_{f, \mathcal{C}}(x, y)=m_{R, \mathcal{C}^{\prime}}(\phi(x), \phi(y))$. Hence, we have the following:

Proposition III.35. Let $f: \mathbb{S}^{2} \rightarrow \mathbb{S}^{2}$ be an expanding Thurston map with no periodic critical points. If there exists $c>0$ such that $D_{n} \geq c(\operatorname{deg} f)^{n / 2}$ for all $n>0$, then $f$ is topologically conjugate to a rational map $R: \widehat{\mathbb{C}} \rightarrow \widehat{\mathbb{C}}$. In addition, there is a visual metric $d$ on $\widehat{\mathbb{C}}$ with expansion factor $\Lambda=(\operatorname{deg}(R))^{1 / 2}$ such that $(\widehat{\mathbb{C}}, d)$ is Ahlfors 2-regular.

Corollary 18.4 in [3] says that:

Lemma III.36. If $d$ is a visual metric for an expanding rational Thurston map $R$, then the identity map id: $\left(\mathbb{S}^{2}, d\right) \rightarrow\left(\mathbb{S}^{2}, \delta\right)$ is a quasisymmetry, where $\delta$ is the chordal metric.

To state our next lemma, let us recall some definitions on metric spaces. We refer the reader to [10] for more details. Given a real-valued function $u$ on a metric space $X$, a Borel function $\rho: X \rightarrow[0, \infty]$ is said to be an upper gradient of $u$ if

$$
|u(x)-u(y)| \leq \int_{\gamma} \rho d s
$$

for each rectifiable curve $\gamma$ joining $x$ and $y$ in $X$. If $u$ is a smooth function on $\mathbb{R}^{n}$, then its gradient $|\nabla u|$ is an upper gradient. We say that a metric space $X$ equipped with a (Borel) measure $\mu$ admits a weak $(1, p)$-Poincaré inequality if there are constants $0<\lambda \leq 1$ and $C \geq 1$ such that for all balls $B$ in $X$, for all bounded continuous functions $u$ on $B$, and for all upper gradients $\rho$ of $u$ on $B$, we have that

$$
\frac{1}{\lambda \mu(B)} \int_{\lambda B}\left|u-u_{\lambda B}\right| d \mu \leq C(\operatorname{diam} B)\left(\frac{1}{\mu(B)} \int_{B} \rho^{p} d \mu\right)^{1 / p},
$$


where $u_{\lambda B}=\frac{1}{\lambda \mu(B)} \int_{\lambda B} u d \mu$. Corollary 7.13 in [10] states that:

Theorem III.37 (Heinonen-Koskela 1998). For two locally compact Q-regular spaces $X$ and $Y$ with $X$ satisfying a weak $(1, p)$-Poincarè inequality for $p<Q$, if $g$ is a quasisymmetric map from $X$ to $Y$, then $g$ and its inverse are absolutely continuous with respect to the Hausdorff Q-measure (of each individual space).

To formulate the next theorem, we call a metric space $X$ linearly locally contractible if there is a $C \geq 1$ so that, for each $x \in X$ and $R<C^{-1} \operatorname{diam}(X)$, the ball $B(x, R)$ can be contracted to a point in $B(x, C R)$. Theorem 6.11 in [10] states that:

Theorem III.38 (Heinonen-Koskela 1998). Let $X$ be a connected and n-regular metric space that is also an orientable $n$-manifold, with $n \geq 2$. If $X$ is linearly locally contractible, then $X$ admits a $(1, p)$-Poincarè inequality for all $p \geq 1$.

Theorem III.39. Let $f: \mathbb{S}^{2} \rightarrow \mathbb{S}^{2}$ be an expanding Thurston map with no periodic critical points. If there exists $c>0$, such that $D_{n} \geq c(\operatorname{deg} f)^{n / 2}$ for all $n>0$, then $f$ is topologically conjugate to a Lattès map.

Proof. By Proposition III.35, there exists a rational function $R$ conjugate to $f$, and $R$ has a visual metric $d$ with expansion factor $\Lambda=\operatorname{deg}(f)^{1 / 2}$ such that $\left(\mathbb{S}^{2}, d\right)$ is Ahlfors 2-regular. Applying Lemma III.36 to the rational map $R$, the identity map id: $(\widehat{\mathbb{C}}, d) \rightarrow(\widehat{\mathbb{C}}, \delta)$ is a quasisymmetry, where $\delta$ is the chordal metric.

The standard Riemann sphere with chordal metric $(\widehat{\mathbb{C}}, \delta)$ satisfies a weak $(1,1)$ Poincaré inequality, with $p=1, Q=2$ by Theorem III.38. By Lemma III.37, the (normalized) Hausdorff measure $H_{d}$ of the metric $d$ and the (normalized) Hausdorff measure $H_{\delta}$ of the metric $\delta$ are mutually absolutely continuous with each other. This implies that a set $E \subset \mathbb{S}^{2}$ has full measure under $H_{\delta}$ if and only if $E$ has full measure under $H_{d}$. 
The dimension of the Lebesgue measure $\Delta$ (i.e., the normalized spherical measure of $\widehat{\mathbb{C}}$ ) with respect to the metric $d$ is

$$
\begin{aligned}
\operatorname{dim}(\Delta, d) & =\inf \left\{\operatorname{dim}_{H_{d}}(E): \Delta(E)=1\right\} \\
& =\inf \left\{\operatorname{dim}_{H_{d}}(E): H_{\delta}(E)=1\right\} \\
& =\inf \left\{\operatorname{dim}_{H_{d}}(E): H_{d}(E)=1\right\}=2 .
\end{aligned}
$$

Theorem II.13 says that the dimension $\operatorname{dim}(\Delta, d)$ of the Lebesgue measure $\Delta$ with respect to the metric $d$ is equal to 2 if and only if $R$ is a Lattès map. Hence, $R$ is a Lattès map and $f$ is topologically conjugate to a Lattès map.

In the proof of Theorem III.39, we also proved that

Corollary III.40. Let $f: \mathbb{S}^{2} \rightarrow \mathbb{S}^{2}$ be an expanding Thurston map with no periodic critical points. If there exists a visual metric on $f$ with expansion factor $\Lambda=\operatorname{deg}(f)^{1 / 2}$, then $f$ is topologically conjugate to a Lattès map.

\subsection{Conclusion}

We get the following topological characterization of Lattès maps:

Theorem III.41. A map $f: \mathbb{S}^{2} \rightarrow \mathbb{S}^{2}$ is topologically conjugate to a Lattès map if and only if the following are true:

- $f$ is an expanding Thurston map;

- $f$ has no periodic critical points;

- there exists $c>0$, such that $D_{n} \geq c(\operatorname{deg} f)^{n / 2}$ for all $n>0$.

Proof. Since all three conditions are preserved under topological conjugacy, we only need to check them for Lattès maps. If $f$ is a Lattès map, then $f$ is an expanding 
Thurston map without periodic critical points. In addition, by Proposition III.18, there exists $c>0$, such that $D_{n} \geq c(\operatorname{deg} f)^{n / 2}$ for all $n>0$.

The sufficiency of the three conditions follows from Theorem III.39.

Corollary III.42. A map $f: \mathbb{S}^{2} \rightarrow \mathbb{S}^{2}$ is topologically conjugate to a Lattès map if and only if the followings are true:

- $f$ is an expanding Thurston map;

- $f$ has no periodic critical points;

- there exists a visual metric on $\mathbb{S}^{2}$ with respect to $f$ with expansion factor $\Lambda=$ $\operatorname{deg}(f)^{1 / 2}$.

Proof. The sufficiency of these conditions follows directly from Corollary III.40.

If $f$ is topologically conjugate to a Lattès map, then $f$ satisfies the three conditions in Theorem III.41. By Proposition III.31, there exists a visual metric on $\mathbb{S}^{2}$ with expansion factor $\Lambda=\operatorname{deg}(f)^{1 / 2}$. 


\section{CHAPTER IV}

\section{Thurston Maps and Asymptotic Upper Curvature}

\subsection{Tile Graphs}

In this section, we construct graphs for expanding Thurston maps. We prove that these graphs are Gromov hyperbolic and their boundary at infinity can be identified with $\mathbb{S}^{2}$.

Let $f: \mathbb{S}^{2} \rightarrow \mathbb{S}^{2}$ be an expanding Thurston map, and $\mathcal{C} \subset \mathbb{S}^{2}$ be a Jordan curve such that $\operatorname{post}(f) \subset \mathcal{C}$. Recall that there is a natural sequence of cell decompositions $\mathcal{D}^{n}(f, \mathcal{C})$ on $\mathbb{S}^{2}$ whose 1 -skeletons are the pull-backs of the Jordan curve $\mathcal{C}$ under $f^{n}$ (see Section 2). Proposition 8.9 in [3] states that there exists a visual metric $d$ for $f$ with expansion factor $\Lambda$ for some $\Lambda>1$.

We define a graph by the cell decompositions of $(f, \mathcal{C})$ as follows. See the discussion about graph theory before Theorem III.21. Let

$$
V=V(f, \mathcal{C})
$$

be the set of all tiles in the cell decompositions $\mathcal{D}^{n}(f, \mathcal{C})$ of $(f, \mathcal{C})$ for $n \geq-1$, where $\mathcal{D}^{-1}(f, \mathcal{C})$ contains a single $(-1)$-tile $\mathbb{S}^{2}$. Let $V$ be the set of vertices of the graph. Define the edge set $E$ as follows: there is an edge between the two vertices $X^{n}, Y^{m} \in V$, denoted $X^{n} \sim Y^{m}$ if as underlying tiles

$$
|n-m| \leq 1 \text { and } X^{n} \cap Y^{m} \neq \emptyset \text {. }
$$


We call the graph

$$
\mathcal{G}(f, \mathcal{C}):=G(V, E)
$$

the tile graph of $(f, \mathcal{C})$. We usually drop one or both parameters in $\mathcal{G}(f, \mathcal{C})$ if $f$ or $\mathcal{C}$ is clear from the context. We call

$$
\ell: V \rightarrow \mathbb{Z}
$$

the level function, where for an $n$-tile $X^{n}$, we have $\ell\left(X^{n}\right)=n$.

If $X \cap Y=\emptyset$, let

$$
\begin{array}{r}
\bar{m}_{f, \mathcal{C}}(X, Y):=\max \left\{m \in \mathbb{N}_{-1}: \text { there exist non-disjoint } m \text {-tiles } X^{m}\right. \text { and } \\
\left.Y^{m} \text {, such that } X \cap X^{m} \neq \emptyset, Y \cap Y^{m} \neq \emptyset\right\} ;
\end{array}
$$

if $X \cap Y \neq \emptyset$, let

$$
\bar{m}_{f, \mathcal{C}}(X, Y):=\min \{\ell(X), \ell(Y)\} .
$$

For $X, Y \in \mathcal{G}$, define

$$
m(X, Y)=m_{f, \mathcal{C}}(X, Y)=\min \left\{\ell(X), \ell(Y), \bar{m}_{f, \mathcal{C}}(X, Y)\right\}
$$

The tile graph $\mathcal{G}$ is path connected since any tile can be connected to the $(-1)$-tile $\mathbb{S}^{2}$. We give $\mathcal{G}$ the path metric $\eta$. Notice that $\mathcal{G}$ is a geodesic space under this metric. The distance from $X$ to the base point $\mathbb{S}^{2}$ is

$$
\eta\left(X, \mathbb{S}^{2}\right)=\ell(X)+1
$$

For $X, Y \in \mathcal{G}$, denote

$$
\begin{aligned}
(X, Y) & :=(X, Y)_{X^{-1}}=(X, Y)_{\mathbb{S}^{2}} \\
& =1 / 2\left[\eta\left(X, \mathbb{S}^{2}\right)+\eta\left(Y, \mathbb{S}^{2}\right)-\eta(X, Y)\right] \\
& =1 / 2[\ell(X)+\ell(Y)-\eta(X, Y)]+1,
\end{aligned}
$$


the Gromov product of $X$ and $Y$ with respect to $X^{-1}=\mathbb{S}^{2}$.

In the following, we are going to prove that the tile graph $\mathcal{G}$ equipped with the path metric $\eta$ is a Gromov hyperbolic space.

Lemma IV.1. There exists a constant $C>1$ such that for any tiles $X, Y \in \mathcal{G}$,

$$
\frac{1}{C} \Lambda^{-m(X, Y)} \leq \operatorname{diam}(X \cup Y) \leq 4 C \Lambda^{-m(X, Y)}
$$

Here and in the following, the diameter function $\operatorname{diam}(\cdot)$ is with respect to the visual metric $d$ on $\mathbb{S}^{2}$.

Proof. Let $m=m(X, Y)$, and let $X^{m}, Y^{m}$ be non-disjoint $m$-tiles such that

$$
X \cap X^{m} \neq \emptyset \text { and } Y \cap Y^{m} \neq \emptyset \text {. }
$$

We have that

$$
\begin{aligned}
\operatorname{diam}(X \cup Y) & \leq \operatorname{diam}(X)+\operatorname{diam}\left(X^{m}\right)+\operatorname{diam}\left(Y^{m}\right)+\operatorname{diam}(Y) \\
& \leq 4 C \Lambda^{-m} .
\end{aligned}
$$

Let $\bar{m}=\bar{m}_{f, \mathcal{C}}(X, Y)$, and let $X^{\bar{m}+1}, Y^{\bar{m}+1}$ be disjoint $(\bar{m}+1)$-tiles such that

$$
X \cap X^{\bar{m}+1} \neq \emptyset, \quad Y \cap Y^{\bar{m}+1} \neq \emptyset .
$$

Then

$$
\begin{aligned}
\operatorname{diam}(X \cup Y) & \geq \max \left\{\operatorname{diam}(X), \operatorname{diam}(Y), d\left(X^{\bar{m}+1}, Y^{\bar{m}+1}\right)\right\} \\
& \geq \frac{1}{C} \max \left\{\Lambda^{-\ell(X)}, \Lambda^{-\ell(Y)}, \Lambda^{-\bar{m}}\right\} \\
& \geq \frac{1}{C} \Lambda^{-\min \{\ell(X), \ell(Y), \bar{m}\}} \\
& =\frac{1}{C} \Lambda^{-m}
\end{aligned}
$$

where $C>1$ is the same $C$ as in Lemma 2.12, which only depends on $f$. 
Lemma IV.2. There exists a constant $k \geq 1$ such that for any tiles $X, Y \in \mathcal{G}$,

$$
\operatorname{diam}(X \cup Y) \leq k \Lambda^{-(X, Y)}
$$

Proof. Let $\eta=\eta(X, Y)$. Pick any path $X_{0}=X, X_{1}, \ldots, X_{\eta+1}=Y$. Then

$$
\begin{aligned}
\operatorname{diam}(X \cup Y) & \leq \sum_{i=0}^{\eta+1} \operatorname{diam}\left(X_{i}\right) \\
& \leq C \sum_{i=0}^{\eta+1} \Lambda^{-\ell\left(X_{i}\right)} \\
& \leq C \min _{0 \leq l \leq \eta+1}\left\{\sum_{i=0}^{l} \Lambda^{-\ell(X)+i}+\sum_{i=l+1}^{\eta+1} \Lambda^{-\ell(Y)+(\eta+1-i)}\right\} \\
& \leq \frac{C \Lambda}{\Lambda-1} \min _{0 \leq l \leq \eta+1}\left\{\Lambda^{-\ell(X)+l}+\Lambda^{-\ell(Y)+(\eta+1-l)}\right\} .
\end{aligned}
$$

Notice that on the right hand-side the minimum is obtained when the two exponents of $\Lambda$ are the same:

$$
-\ell(X)+l=-\ell(Y)+(\eta+1-l),
$$

so we let

$$
l=\left[\frac{1}{2}(\ell(X)-\ell(Y)+\eta)\right]+1
$$

be the integer part of $\frac{1}{2}(\ell(X)-\ell(Y)+\eta)+1$. Hence, we have

$$
\begin{aligned}
\operatorname{diam}(X \cup Y) & \leq \frac{2 C \Lambda}{\Lambda-1} \Lambda^{-[1 / 2(\ell(X)+\ell(Y)-\eta)]+1} \\
& \leq k \Lambda^{-(X, Y)}
\end{aligned}
$$

where $C>1$ is the same $C$ as in Lemma 2.12 , and

$$
k=\frac{2 C \Lambda^{3}}{\Lambda-1}
$$

also only depends on $f$.

Proposition IV.3. There exists a constant $C^{\prime}>0$, such that for any tiles $X, Y \in \mathcal{G}$,

$$
m(X, Y)-1 \leq(X, Y) \leq m(X, Y)+C^{\prime} .
$$


Proof. By Lemma IV.1 and Lemma IV.2, we have that

$$
\frac{1}{C} \Lambda^{-m(X, Y)} \leq \operatorname{diam}(X \cup Y) \leq k \Lambda^{-(X, Y)}
$$

for some constants $C, k>1$ which only depend on $f$. Hence, there exists a constant $C^{\prime}>0$, such that for any tiles $X, Y \in \mathcal{G}$,

$$
(X, Y) \leq m(X, Y)+C^{\prime} .
$$

For the other inequality, let $m=m(X, Y)$, and let $X^{m}, Y^{m}$ be non-disjoint $m$-tiles such that

$$
X \cap X^{m} \neq \emptyset, \quad Y \cap Y^{m} \neq \emptyset .
$$

So

$$
\eta\left(X, X^{m}\right) \leq \ell(X)-m+1
$$

and

$$
\eta\left(Y, Y^{m}\right) \leq \ell(Y)-m+1 .
$$

By the triangle inequality, we have that

$$
\begin{aligned}
\eta(X, Y) & \leq \eta\left(X, X^{m}\right)+\eta\left(X^{m}, Y\right) \\
& \leq \eta\left(X, X^{m}\right)+\eta\left(Y^{m}, Y\right)+1 \\
& \leq(\ell(X)-m)+(\ell(Y)-m)+3 .
\end{aligned}
$$

Hence, we obtain that

$$
\begin{aligned}
(X, Y) & =(X, Y)_{X^{-1}}=1 / 2[\ell(X)+\ell(Y)-\eta(X, Y)]+1 \\
& \geq 1 / 2[\ell(X)+\ell(Y)-(\ell(X)-m)-(\ell(Y)-m)-3]+1 \\
& \geq m(X, Y)-1
\end{aligned}
$$


Lemma IV.4. There exists a number $c \geq 0$ such that for any tiles $X, Y, Z \in \mathcal{G}$,

$$
m(X, Y) \geq \min \{m(X, Z), m(Y, Z)\}-c
$$

Proof. For any $X, Y, Z \in \mathcal{G}$,

$$
\begin{aligned}
& \operatorname{diam}(X \cup Y)= \max \left\{d(x, y), d\left(x, x^{\prime}\right), d\left(y, y^{\prime}\right): x, x^{\prime} \in X, y, y^{\prime} \in Y\right\} \\
& \leq \max \left\{d(x, z)+d(z, y), d\left(x, x^{\prime}\right), d\left(y, y^{\prime}\right):\right. \\
&\left.x, x^{\prime} \in X, y, y^{\prime} \in Y, z \in Z\right\} \\
& \leq \quad \max \left\{d(x, z), d\left(x, x^{\prime}\right): x \in X, z \in Z\right\} \\
& \quad+\max \left\{d(z, y), d\left(y, y^{\prime}\right): y \in Y, z \in Z\right\} \\
& \leq \operatorname{diam}(X \cup Z)+\operatorname{diam}(Z \cup Y),
\end{aligned}
$$

and so

$$
\operatorname{diam}(X \cup Y) \leq 2 \max \{\operatorname{diam}(X \cup Z), \operatorname{diam}(Z \cup Y)\}
$$

By Lemma IV.1, there exists a constant $k>1$, such that for any $X, Y \in \mathcal{G}$,

$$
\frac{1}{k} \Lambda^{-m(X, Y)} \leq \operatorname{diam}(X \cup Y) \leq k \Lambda^{-m(X, Y)}
$$

Hence, by the inequalities above and inequality (4.3), we have that

$$
\begin{aligned}
m(X, Y) & \geq-\log _{\Lambda}(k \operatorname{diam}(X \cup Y)) \\
& \geq-\log _{\Lambda}(2 k \max \{\operatorname{diam}(X \cup Z), \operatorname{diam}(Z \cup Y)\}) \\
& \geq \min \left\{-\log _{\Lambda}(2 k \operatorname{diam}(X \cup Z)),-\log _{\Lambda}(2 k \operatorname{diam}(Z \cup Y))\right\} \\
& \geq \min \{m(X, Z), m(Y, Z)\}-c
\end{aligned}
$$

for some $c \geq 0$ that only depends on $f$. 
Theorem IV.5. Let $f: \mathbb{S}^{2} \rightarrow S^{2}$ be an expanding Thurston map without periodic critical points and let $\mathcal{C} \subset \mathbb{S}^{2}$ be a Jordan curve containing post $(f)$. Then the tile graph $\mathcal{G}(f, \mathcal{C})$ equipped with the path metric $\eta$ is a Gromov hyperbolic space.

Proof. For any tiles $X, Y \in \mathcal{G}$, by Proposition IV.3, the Gromov product $(X, Y)$ defined in equation (4.2) is equal to $m(X, Y)$ up to a constant which only depends on $f$. So by Lemma IV.4, there exists a constant $c^{\prime}>0$, such that for any tiles $X, Y, Z \in \mathcal{G}$,

$$
(X, Y) \geq \min \{(X, Z),(Y, Z)\}-c^{\prime}
$$

Therefore, the graph $G(f, \mathcal{C})$ equipped with the path metric $\eta$ is a Gromov hyperbolic space.

Remark 4. In the proofs of Proposition IV.3 and Lemma IV.4, we used visual metrics as a bridge to connect $m(\cdot, \cdot)$ and the Gromov product $(\cdot, \cdot)$. This idea is contained in [4]. Theorem IV.5, Proposition IV.3 and Lemma IV.4 can also be proved combinatorially without using visual metrics.

Proposition IV.6. For any Jordan curves $\mathcal{C}$ and $\mathcal{C}^{\prime}$ containing post $(f)$, the tile graphs $\mathcal{G}=\mathcal{G}(f, \mathcal{C})$ and $\mathcal{G}^{\prime}=\mathcal{G}\left(f, \mathcal{C}^{\prime}\right)$ equipped with path metric respectively are rough-isometric.

Proof. By equation (4.2), for any $X, Y \in \mathcal{G}(f, \mathcal{C})$, we have

$$
\eta(X, Y)=\ell(X)+\ell(Y)+2-2(X, Y)
$$

We have similar relations for the path metric $\eta^{\prime}$ of $\mathcal{G}^{\prime}$. Let $m=m_{f, \mathcal{C}}$ and $m^{\prime}=m_{f, \mathcal{C}^{\prime}}$ as defined in equation (4.1). We know that $m(X, Y)$ and $(X, Y)$ are equal up to a constant that only depends on $f$ by Proposition IV.3. So if we can show that there exists a bijection $g: \mathcal{G} \rightarrow \mathcal{G}^{\prime}$ and a constant $\lambda \geq 0$, such that for any $X, Y \in \mathcal{G}$

$$
m(X, Y)-\lambda \leq m^{\prime}(g(X), g(Y)) \leq m(X, Y)+\lambda,
$$


then by equation (4.4), the map $g$ will be a rough isometry between the path metrics of $\mathcal{G}$ and $\mathcal{G}^{\prime}$.

Fix $p \in \operatorname{post}(f)$, and define

$$
g: \mathcal{G}(f, \mathcal{C}) \rightarrow \mathcal{G}\left(f, \mathcal{C}^{\prime}\right)
$$

by defining a bijection between $n$-tiles of $(f, \mathcal{C})$ and $\left(f, \mathcal{C}^{\prime}\right)$ for all $n \geq-1$. For $n=-1$, let

$$
g\left(\mathbb{S}^{2}\right)=\mathbb{S}^{2}
$$

For $n \geq 0$, and for any $q \in f^{-n}(p)$, we claim that there exists a bijection $g_{n, q}$ between $n$-tiles of $(f, \mathcal{C})$ containing $q$ and $n$-tiles of $\left(f, \mathcal{C}^{\prime}\right)$ containing $q$,

$$
g_{n, q}:\left\{n \text {-tile } X \in \mathcal{D}^{n}(f, \mathcal{C}): q \in X\right\} \rightarrow\left\{n \text {-tile } X^{\prime} \in \mathcal{D}^{n}\left(f, \mathcal{C}^{\prime}\right): q \in X^{\prime}\right\}
$$

Indeed, the number of tiles containing $q$ is equal to the degree of $f^{n}$ at $q$, and this justifies the existence of the bijection $g_{n, q}$. Since every $n$-tile contains exactly one point in $f^{-n}(p)$, we get a bijection of all $n$-tiles by $g_{n, q}$ for $q \in f^{-n}(p)$.

For any $X, Y \in \mathcal{G}$, let $X^{\prime}, Y^{\prime} \in \mathcal{G}^{\prime}$ be their images under $g$. It follows from the definition of $g$ that

$$
X \cap X^{\prime} \neq \emptyset \text { and } Y \cap Y^{\prime} \neq \emptyset \text {. }
$$

Now we are going to show that there exists $k \geq 1$, such that for any $X, Y \in \mathcal{G}$,

$$
\frac{1}{k} \operatorname{diam}\left(X^{\prime} \cup Y^{\prime}\right) \leq \operatorname{diam}(X \cup Y) \leq k \operatorname{diam}\left(X^{\prime} \cup Y^{\prime}\right) .
$$

Let $m=m(X, Y)$. We have that

$$
\begin{aligned}
\operatorname{diam}(X \cup Y) & \leq \operatorname{diam}(X)+\operatorname{diam}\left(X^{\prime} \cup Y^{\prime}\right)+\operatorname{diam}(Y) \\
& \leq C^{2} \operatorname{diam}\left(X^{\prime}\right)+C^{2} \operatorname{diam}\left(Y^{\prime}\right)+\operatorname{diam}\left(X^{\prime} \cup Y^{\prime}\right) \\
& \leq\left(C^{2}+1\right) \operatorname{diam}\left(X^{\prime} \cup Y^{\prime}\right),
\end{aligned}
$$


where $C>1$ is the same $C$ as in Lemma 2.12, which only depends on $f$. This implies that

$$
\operatorname{diam}(X \cup Y) \leq k \operatorname{diam}\left(X^{\prime} \cup Y^{\prime}\right)
$$

for some $k>1$ only depending on $f$. Similarly, we get that

$$
\operatorname{diam}\left(X^{\prime} \cup Y^{\prime}\right) \leq k \operatorname{diam}(X \cup Y)
$$

Since $\operatorname{diam}(X \cup Y)$ and $\Lambda^{-m(X, Y)}$ are the same up to a scaling by Lemma IV.1, there exists a constant $\lambda>0$, such that

$$
m(X, Y)-\lambda \leq m^{\prime}(g(X), g(Y)) \leq m(X, Y)+\lambda
$$

for all $X, Y \in \mathcal{G}(f, \mathcal{C})$.

Remark 5. In the proof of Proposition IV.6, the bijective rough-isometry $g$ between tile graphs of two different Jordan curves induces a bijection $g_{\infty}$ on the boundary at infinity of these two tile graphs.

Proposition IV.7. The boundary at infinity $\partial_{\infty} \mathcal{G}$ of a graph tile $\mathcal{G}(f, \mathcal{C})$ can be identified with $\mathbb{S}^{2}$. Under this identification, a metric d is a visual metric on $\mathbb{S}^{2}$ with respect to the expanding Thurston map $f$ if and only if $d$ is a visual metric (in the sense of Gromov hyperbolic space) on $\partial_{\infty} \mathcal{G}$.

Here the metric $d$ on $\partial_{\infty} \mathcal{G}$ means the pull-pack metric of $d$ under the identification.

Proof. Let $d$ be a visual metric with expansion factor $\Lambda$ of $\mathbb{S}^{2}$ with respect to $f$.

For any converging sequence $\left\{X_{n}\right\}$

$$
\lim _{i, j \rightarrow \infty}\left(X_{i}, X_{j}\right)=\infty
$$

we have a filtration

$$
\bigcup_{i=1}^{\infty} X_{i} \supset \bigcup_{i=2}^{\infty} X_{i} \supset \ldots \supset \bigcup_{i=n}^{\infty} X_{i} \supset \bigcup_{i=n+1}^{\infty} X_{i} \supset \ldots
$$


with

$$
\operatorname{diam}\left(\bigcup_{i=n}^{\infty} X_{i}\right) \rightarrow 0 \text { as } n \rightarrow \infty .
$$

Hence, there exists a limit point $x \in \mathbb{S}^{2}$ such that for any $\epsilon>0$, there exists $N>0$ such that for all $n>N$,

$$
\bigcup_{i=n}^{\infty} X_{i} \subset N_{\epsilon}(x),
$$

where $N_{\epsilon}(x)$ is an $\epsilon$-neighborhood of $x$ in $\mathbb{S}^{2}$, i.e.,

$$
X_{n} \subset N_{\epsilon}(x),
$$

or

$$
d\left(x, X_{n}\right)<\epsilon
$$

We claim that the limit point is unique. Indeed, if there exists $y \in \mathbb{S}^{2}$ also satisfying (4.5), then

$$
d(x, y) \leq d\left(x, \operatorname{diam}\left(X_{n}\right)\right)+d\left(y, \operatorname{diam}\left(X_{n}\right)\right) \rightarrow 0 \text { as } n \rightarrow \infty .
$$

Hence, $x=y$ and the claim follows. Let $\left\{Y_{n}\right\}$ be a converging sequence equivalent to $\left\{X_{i}\right\}$, i.e.,

$$
\lim _{i \rightarrow \infty}\left(X_{i}, Y_{i}\right)=\infty
$$

We claim that the limit point of $\left\{Y_{n}\right\}$ is $x$. Indeed, by Lemma IV.2, we have

$$
d\left(x, Y_{n}\right) \leq d\left(x, X_{n}\right)+d\left(Y_{n}, X_{n}\right) \leq d\left(x, X_{n}\right)+k \Lambda^{-\left(Y_{n}, X_{n}\right)} \rightarrow 0
$$

as $n$ goes to infinity since $\left(Y_{n}, X_{n}\right) \rightarrow \infty$. Hence, any equivalent converging sequences have the same limit point, and we can assign a limit point to an equivalence class of converging sequences. 
We define

$$
h: \partial_{\infty} \mathcal{G} \rightarrow \mathbb{S}^{2}
$$

by mapping any equivalence class of converging sequences to its limit point. For any $x \in \mathbb{S}^{2}$, there exists $X_{i}$ with $\ell\left(X_{i}\right)=i$ containing $x$, for any $i \geq-1$. Then by Lemma IV.2, we have that

$$
\begin{aligned}
\left(X_{i}, X_{j}\right) & \leq-\log _{\Lambda} \operatorname{diam}\left(X_{i} \cup X_{j}\right)+\log k \\
& \leq-\log _{\Lambda}\left(\min \left\{\operatorname{diam}\left(X_{i}\right), \operatorname{diam}\left(X_{j}\right)\right\}\right)+\log k \rightarrow \infty
\end{aligned}
$$

as $i, j \rightarrow \infty$, where $k \geq 1$ is a constant as in Lemma IV.2. So $\left\{X_{i}\right\}$ is a converging sequence with limit point $x$. Hence, the map $h$ is surjective. For any two converging non-equivalent sequences $\left\{X_{i}\right\}$ and $\left\{Y_{i}\right\}$, let $x$ and $y$ be their limit points respectively. If $x=y$, then

$$
\operatorname{diam}\left(X_{n} \cup Y_{n}\right) \rightarrow 0 \text { as } n \rightarrow \infty
$$

So by Lemma IV.1 and IV.3,

$$
\left(X_{n}, Y_{n}\right) \geq m\left(X_{n}, Y_{n}\right)-1 \geq-\log _{\Lambda}\left(C \operatorname{diam}\left(X_{n} \cup Y_{n}\right)\right)-1 \rightarrow \infty
$$

as $n$ goes to infinity, which is a contradiction to the assumption of non-equivalence of $\left\{X_{i}\right\}$ and $\left\{Y_{i}\right\}$. Hence, we have that $x \neq y$ and $h$ is injective.

We only need to show that that there exists a constant $C>0$ such that for any $\xi, \xi^{\prime} \in \partial_{\infty} \mathcal{G}, x=h(\xi)$ and $y=h\left(\xi^{\prime}\right)$

$$
\frac{1}{C} \Lambda^{-\left(\xi, \xi^{\prime}\right)} \leq d(x, y) \leq 4 C \Lambda^{-\left(\xi, \xi^{\prime}\right)}
$$

Pick any $\left\{X_{n}\right\} \in \xi$ and $\left\{Y_{n}\right\} \in \xi^{\prime}$. By Lemma IV.1

$$
\frac{1}{C} \Lambda^{-\left(X_{n}, Y_{n}\right)} \leq \operatorname{diam}\left(X_{n} \cup Y_{n}\right) \leq 4 C \Lambda^{-\left(X_{n}, Y_{1}\right.} .
$$


Taking the supremum limit, we get

$$
\frac{1}{C} \limsup _{n \rightarrow \infty} \Lambda^{-\left(X_{n}, Y_{n}\right)} \leq \lim _{n \rightarrow \infty} \operatorname{diam}\left(X_{n} \cup Y_{n}\right) \leq 4 C \limsup _{n \rightarrow \infty} \Lambda^{-\left(X_{n}, Y_{n}\right)} .
$$

Hence, we have

$$
\begin{aligned}
\frac{1}{C} \Lambda^{-\liminf _{n \rightarrow \infty}\left(X_{n}, Y_{n}\right)} \leq \lim _{n \rightarrow \infty} \operatorname{diam}( & \left.X_{n} \cup Y_{n}\right) \\
& \leq 4 C \Lambda^{-\liminf _{n \rightarrow \infty}\left(X_{n}, Y_{n}\right)}
\end{aligned}
$$

Since

$$
d(x, y)=\lim _{n \rightarrow \infty} \operatorname{diam}\left(X_{n}, Y_{n}\right)
$$

and

$$
\left(\xi, \xi^{\prime}\right) \leq \liminf _{n \rightarrow \infty}\left(X_{n}, Y_{n}\right)
$$

by inequality (4.6)

$$
\begin{aligned}
d(x, y) & =\lim _{n \rightarrow \infty} \operatorname{diam}\left(X_{n} \cup Y_{n}\right) \\
& \leq 4 C \Lambda^{-\liminf _{n \rightarrow \infty}\left(X_{n}, Y_{n}\right)} \\
& \leq 4 C \Lambda^{-\left(\xi, \xi^{\prime}\right)} .
\end{aligned}
$$

Since

$$
\left(\xi, \xi^{\prime}\right)=\inf \liminf _{n \rightarrow \infty}\left(X_{n}, Y_{n}\right)
$$

where infimum is taken for all $\left\{X_{n}\right\} \in \xi$ and $\left\{Y_{n}\right\} \in \xi^{\prime}$, by inequality (4.6),

$$
\frac{1}{C} \sup \Lambda^{-\liminf _{n \rightarrow \infty}\left(X_{n}, Y_{n}\right)} \leq \lim _{n \rightarrow \infty} \operatorname{diam}\left(X_{n} \cup Y_{n}\right)=d(x, y)
$$

where supremum is taken for all $\left\{X_{n}\right\} \in \xi$ and $\left\{Y_{n}\right\} \in \xi^{\prime}$. Hence,

$$
\frac{1}{C} \Lambda^{-\left(\xi, \xi^{\prime}\right)}=\frac{1}{C} \Lambda^{-\inf \liminf _{n \rightarrow \infty}\left(X_{n}, Y_{n}\right)} \leq d(x, y) .
$$

Combining equations (4.7) and (4.8), we get that

$$
\frac{1}{C} \Lambda^{-\left(\xi, \xi^{\prime}\right)} \leq d(x, y) \leq 4 C \Lambda^{-\left(\xi, \xi^{\prime}\right)},
$$




$$
\frac{1}{C} \Lambda^{-\left(\xi, \xi^{\prime}\right)} \leq d\left(h(\xi), h\left(\xi^{\prime}\right)\right) \leq 4 C \Lambda^{-\left(\xi, \xi^{\prime}\right)}
$$

for all $\xi, \xi^{\prime} \in \partial_{\infty} \mathcal{G}$. Therefore, the push-pack of the metric $d$ on $\mathbb{S}^{2}$ under $h$ is a visual metric on $\partial_{\infty} \mathcal{G}$.

Since $d$ is a visual metric with respect to $f$, equation (4.9) implies that there exists a constant $c \geq 0$ such that for all $x, y \in \mathbb{S}^{2}$, and $\xi=h^{-1}(x), \xi=h^{-1}(y)$,

$$
\left(\xi, \xi^{\prime}\right)-c \leq m(x, y) \leq\left(\xi, \xi^{\prime}\right)+c
$$

Let $\rho$ be a visual metric on $\partial_{\infty} \mathcal{G}$ on the Gromov hyperbolic space, so there exists constant $k \geq 1$, such that for any $\xi, \xi^{\prime} \in \partial_{\infty} \mathcal{G}$

$$
\frac{1}{k} \Lambda^{-\left(\xi, \xi^{\prime}\right)} \leq \rho\left(\xi, \xi^{\prime}\right) \leq k \Lambda^{-\left(\xi, \xi^{\prime}\right)}
$$

By equation (4.10), there exists a constant $k^{\prime} \geq 1$, such that

$$
\frac{1}{k^{\prime}} \Lambda^{-(x, y)} \leq \frac{1}{k} \Lambda^{-\left(\xi, \xi^{\prime}\right)} \leq \rho\left(h^{-1}(x), h^{-1}(y)\right) \leq k \Lambda^{-\left(\xi, \xi^{\prime}\right)} \leq k^{\prime} \Lambda^{-m(x, y)},
$$

where $x, y \in \mathbb{S}^{2}, \xi=h^{-1}(x)$ and $\xi=h^{-1}(y)$. Therefore, the push-pack of the metric $\rho$ on $\partial_{\infty} \mathcal{G}$ under $h^{-1}$ is a visual metric on $\mathbb{S}^{2}$.

For any Jordan curves $\mathcal{C}$ and $\mathcal{C}^{\prime}$ containing post $(f)$, let $\partial_{\infty} \mathcal{G}=\partial_{\infty} \mathcal{G}(f, \mathcal{C})$ and $\partial_{\infty} \mathcal{G}^{\prime}=\partial_{\infty} \mathcal{G}\left(f, \mathcal{C}^{\prime}\right)$ be the boundary at infinity of the tile graphs $\mathcal{G}(f, \mathcal{C})$ and $\mathcal{G}\left(f, \mathcal{C}^{\prime}\right)$ respectively. By the proposition above, there exist identifications

$$
h: \partial_{\infty} \mathcal{G} \rightarrow \mathbb{S}^{2}
$$

and

$$
h^{\prime}: \partial_{\infty} \mathcal{G}^{\prime} \rightarrow \mathbb{S}^{2} .
$$


So we have the following diagram

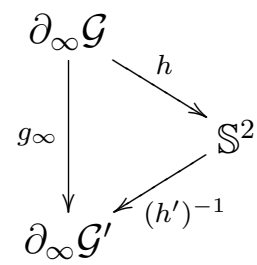

This induced bijection $g_{\infty}=\left(h^{\prime}\right)^{-1} \circ h$ should be the same as $g_{\infty}$ as in the remark after Proposition IV.6. In addition, under this identification, visual metrics on $\partial_{\infty} \mathcal{G}$ and $\partial_{\infty} \mathcal{G}^{\prime}$ are also identified. This is the following corollary.

Corollary IV.8. For any Jordan curves $\mathcal{C}$ and $\mathcal{C}^{\prime}$ containing post $(f)$, there exists a natural identification between $\mathcal{G}=\partial_{\infty} \mathcal{G}(f, \mathcal{C})$ and $\mathcal{G}^{\prime}=\partial_{\infty} \mathcal{G}\left(f, \mathcal{C}^{\prime}\right)$. Under this identification, a metric $\rho$ is a visual metric on $\partial_{\infty} \mathcal{G}$ if and only if it is a visual metric on $\partial_{\infty} \mathcal{G}^{\prime}$.

\subsection{Asymptotic Upper Curvature}

Let $f: \mathbb{S}^{2} \rightarrow S^{2}$ be an expanding Thurston map without periodic critical points. We define the asymptotic upper curvature of $f$ as

$$
K_{u}(f):=K_{u}(\mathcal{G}(f, \mathcal{C}))
$$

where $\mathcal{C} \subset \mathbb{S}^{2}$ is any Jordan curve containing post $(f)$ and $\mathcal{G}=\mathcal{G}(f, \mathcal{C})$ denotes the Gromov hyperbolic graph constructed from the cell decompositions of $(f, \mathcal{C})$. For any Jordan curves $\mathcal{C}$ and $\mathcal{C}^{\prime}$, the Gromov hyperbolic space $\mathcal{G}(f, \mathcal{C})$ and $\mathcal{G}\left(f, \mathcal{C}^{\prime}\right)$ are rough-isomeric by Proposition IV.6, and the asymptotic upper curvature is invariant under rough-isometry, so

$$
K_{u}(\mathcal{G}(f, \mathcal{C}))=K_{u}\left(\mathcal{G}\left(f, \mathcal{C}^{\prime}\right)\right)
$$

Therefore, the asymptotic upper curvature $K(f)$ is well-defined in equation (4.11). 
Theorem IV.9. Let $f: \mathbb{S}^{2} \rightarrow \mathbb{S}^{2}$ be an expanding Thurston map without periodic critical points. The asymptotic upper curvature of $f$ satisfies

$$
K_{u}(f) \geq-\frac{1}{4} \log ^{2}(\operatorname{deg} f)
$$

The tile graph $\mathcal{G}=\mathcal{G}(f)$ is an $A C_{u}(\kappa)$-space with

$$
\kappa=-\frac{1}{4} \log ^{2}(\operatorname{deg} f)
$$

if and only if the map $f$ is topologically conjugate to a Lattès map.

Proof. The first part follows directly from the definition of asymptotic upper curvature of $f$ and from Theorem II.15.

If $f$ is topologically conjugate to a Lattès map, then by Corollary III.42, there exists a visual metric on $\mathbb{S}^{2}$ with respect to $f$ with expansion factor $\Lambda=\operatorname{deg}(f)^{1 / 2}$. By Proposition IV.7, there exists a visual metric on $\partial_{\infty} \mathcal{G}$ in the sense of Gromov hyperbolic space with expansion factor $\Lambda=\operatorname{deg}(f)^{1 / 2}$. By Theorem II.15, the Gromov hyperbolic space $\mathcal{G}$ is an $\mathrm{AC}_{u}(\kappa)$-space with

$$
\kappa=-\frac{1}{4} \log ^{2}(\operatorname{deg} f) .
$$

If $\mathcal{G}$ is an $\mathrm{AC}_{u}(\kappa)$-space with

$$
\kappa=-\frac{1}{4} \log ^{2}(\operatorname{deg} f)
$$

then for all $X, X^{\prime} \in \mathcal{G}$ and all finite sequences $X_{0}=X, X_{1}, \ldots, X_{n}=X^{\prime}$ in $X$,

$$
\left(X, X^{\prime}\right) \geq \min _{i=1,2, \ldots, n}\left(X_{i-1}, X_{i}\right)-\frac{\log n}{\log (\operatorname{deg} f)^{1 / 2}}-c .
$$

Let $D_{n}$ be the minimum $n$-tiles needed to join the opposite sides of Jordan curve $\mathcal{C}$ as defined in, for $n>0$. Let $P_{n}=X_{1} \ldots X_{D_{n}}$ be an $n$-tile chain joining opposite sides of $\mathcal{C}$. By the equation (4.12), we have

$$
\left(X_{1}, X_{D_{n}}\right) \geq \min _{i=1,2, \ldots, D_{n}}\left(X_{i-1}, X_{i}\right)-\frac{\log D_{n}}{\log (\operatorname{deg} f)^{1 / 2}}-c
$$




$$
\frac{\log D_{n}}{\log (\operatorname{deg} f)^{1 / 2}} \geq \min _{i=1,2, \ldots, D_{n}}\left(X_{i-1}, X_{i}\right)-\left(X_{1}, X_{D_{n}}\right)-c
$$

By equation (4.2), we have

$$
\begin{aligned}
\left(X_{i-1}, X_{i}\right) & =\frac{1}{2}\left[\ell\left(X_{i-1}\right)+\ell\left(X_{i}\right)-\eta\left(X_{i-1}, X_{i}\right)\right]+1 \\
& =\frac{1}{2}\left[2 n-\eta\left(X_{i-1}, X_{i}\right)\right]+1 \\
& \geq n+1-\frac{1}{4} N
\end{aligned}
$$

where $N>0$ is a constant only depending on $f$ as in Lemma II.8, i.e. the constant $N$ is the upper bound of the degree of $f^{n}$ at any point in $\mathbb{S}^{2}$. Applying equation (4.14) and Lemma IV.2 to equation (4.13), we have

$$
\begin{aligned}
\frac{\log D_{n}}{\log (\operatorname{deg} f)^{1 / 2}} & \geq \min _{i=1,2, \ldots, D_{n}}\left(X_{i-1}, X_{i}\right)-\left(X_{1}, X_{D_{n}}\right)-c \\
& \geq n+1-\frac{1}{4} N-\log _{\Lambda}\left(\operatorname{diam}\left(X_{1} \cup X_{D_{n}}\right)\right)+\log _{\Lambda} k-c,
\end{aligned}
$$

where $k \geq 1$ only depends on $f$ and $\mathcal{C}$ as in Lemma IV.2, and $N>0$ only depends on $f$. Let $D$ be the maximum of diameter of the two 0-tiles, then

$$
\log _{\Lambda}\left(\operatorname{diam}\left(X_{1} \cup X_{D_{n}}\right)\right) \leq D
$$

So

$$
\begin{aligned}
\frac{\log D_{n}}{\log (\operatorname{deg} f)^{1 / 2}} & \geq n+1-\frac{1}{4} N-\log _{\Lambda}(D)+\log _{\Lambda} k-c \\
& =n+C .
\end{aligned}
$$

Here the constant

$$
\left.C=1-\frac{1}{4} N-\log _{\Lambda}(D)\right)+\log _{\Lambda} k-c
$$


only depends on $f$ and $\mathcal{C}$. Hence, we have

$$
\log D_{n} \geq(n+C) \log (\operatorname{deg} f)^{1 / 2}
$$

Therefore, we have

$$
D_{n} \geq c(\operatorname{deg} f)^{n / 2}
$$

where $c=(\operatorname{deg} f)^{C / 2}$ only depends on $f$ and $\mathcal{C}$. By Theorem III.41, the map $f$ is topologically conjugate to a Lattès map. 
BIBLIOGRAPHY 


\section{BIBLIOGRAPHY}

[1] Mario Bonk and Thomas Foertsch. Asymptotic upper curvature bounds in coarse geometry. Math. Z., 253:753-785, 2006.

[2] Mario Bonk and Bruce Kleiner. Quasisymmetric parametrizations of two-dimensional metric spheres. Invent. Math., 150:127-183, 2002.

[3] Mario Bonk and Daniel Meyer. Expanding Thurston maps. Preprint, 2010. arXiv:1009.3647v1.

[4] Marc Bourdon and Hervé Pajot. Cohomologie $l_{p}$ et espaces de Besov. J. Reine Angew. Math., $558: 85-108,2003$.

[5] Sergei Buyalo and Viktor Schroeder. Elements of asymptotic geometry. EMS Monographs in Mathematics. European Mathematical Society, Zürich, 2007.

[6] Reinhard Diestel. Graph theory, volume 173 of Graduate Texts in Mathematics. SpringerVerlag, Berlin, third edition, 2005.

[7] Adrien Douady and John H. Hubbard. A proof of Thurston's topological characterization of rational functions. Acta Math., 171:263-297, 1993.

[8] Pierre Fatou. Sur les substitutions rationnelles. C. R. Acad. Sci. Paris, 164:806-808, 1917.

[9] Peter Haïssinsky and Kevin M. Pilgrim. Coarse expanding conformal dynamics. Astérisque, 325, 2009.

[10] Juha Heinonen and Pekka Koskela. Quasiconformal maps in metric spaces with controlled geometry. Acta Math., 181:1-61, 1998.

[11] Gaston Julia. Mémoire sur l'iteration des fonctions rationnelles. J. de Math. Pure et Appl., 8:47-245, 1918.

[12] Samuel Lattès. Sur l'iteration des substitutions rationnelles et les fonctions de Poincaré. $C$. R. Acad. Sci. Paris, 166:26-28, 1918.

[13] Peter Lax. Linear algebra and its applications, volume 10 of Pure and applied mathematics. Wiley, New York, second edition, 2008.

[14] Benoit Mandelbrot. Les objets fractals.

[15] Benoit B. Mandelbrot. The fractal geometry of nature.

[16] Ricardo Mañé, Paulo Sad, and Dennis Sullivan. On the dynamics of rational maps. Ann. Sci. École Norm. Sup. (4), 16(2):193-217, 1983.

[17] Daniel Meyer. Dimension of elliptic harmonic measure of snowspheres. Illinois J. Math., 53:691-721, 2009.

[18] John Milnor. On Lattès maps. In Dynamics on the Riemann sphere, pages 9-43. Eur. Math. Soc., Zürich, 2006. 
[19] M. H. A. Newman. Elements of the topology of plane sets of points. Dover Publications Inc., New York, second edition, 1992.

[20] Ernst Schröder. Ueber iterirte Functionen. Math. Ann., 3(2):296-332, 1871.

[21] Dennis Sullivan. Quasiconformal homeomorphisms and dynamics. I. Solution of the FatouJulia problem on wandering domains. Ann. of Math. (2), 122:401-418, 1985.

[22] William Thurston. The geometry and topology of three-manifolds. Princeton University lecture notes.

[23] Anna Zdunik. Parabolic orbifolds and the dimension of the maximal measure for rational maps. Invent. Math., 99:627-649, 1990. 